\title{
Efficient Channel Estimation for Reconfigurable MIMO Antennas: Training Techniques and Performance Analysis
}

\author{
Israfil Bahceci, Member, IEEE, Mehedi Hasan, Tolga M. Duman, Fellow, IEEE, \\ and Bedri A. Cetiner, Member, IEEE
}

\begin{abstract}
Multifunctional and reconfigurable multiple-input multiple-output (MR-MIMO) antennas are capable of dynamically changing the operation frequencies, polarizations, and radiation patterns, and can remarkably enhance system capabilities. However, in coherent communication systems, using MR-MIMO antennas with a large number of operational modes may incur prohibitive complexity due to the need for channel state estimation for each mode. To address this issue, we derive an explicit relation among the radiation patterns for the antenna modes and the resulting channel gains. We propose a joint channel estimation/prediction scheme where only a subset of all the antenna modes is trained for estimation, and then, the channels associated with the modes that are not trained are predicted using the correlations among the different antenna modes. We propose various training mechanisms with reduced overhead and improved estimation performance, and study the impact of channel estimation error and training overhead on the MR-MIMO system performance. We demonstrate that one can achieve significantly improved data rates and lower error probabilities utilizing the proposed approaches. For instance, under practical settings, we observe about $25 \%$ throughput increase or about 3-dB signal-to-noise ratio improvement under the same training overhead with respect to non-reconfigurable antenna systems.
\end{abstract}

Index Terms-Multifunctional and reconfigurable antennas, MIMO, multipath channels, channel estimation, antenna radiation patterns.

\section{INTRODUCTION}

$\mathbf{M}$ ULTIFUNCTIONAL and reconfigurable antennas (MRAs) form a new class of antennas that can dynamically be configured to operate at different frequency bands, and with different polarizations and radiation patterns [1], [2].

Manuscript received January 2, 2016; revised August 18, 2016; accepted October 22, 2016. Date of publication November 8, 2016; date of current version January 6, 2017. This work was supported by AFOSR under Grant FA 9550-15-1-0040 DEF. The work of T. M. Duman was supported by the EC Marie Curie Career Integration under Grant PCIG12-GA-2012-334213. The associate editor coordinating the review of this paper and approving it for publication was K. Haneda.

I. Bahceci, M. Hasan, and B. A. Cetiner are with the Department of Electrical and Computer Engineering, Utah State University, Logan, UT 84322 USA (e-mail: bahceci@usu.edu; mehedi.hasan@usu.edu; bedri.cetiner@usu.edu).

T. M. Duman is with the Department of Electrical and Electronics Engineering, Bilkent University, Ankara 06800, Turkey (e-mail: duman@ee.bilkent.edu.tr).

Color versions of one or more of the figures in this paper are available online at http://ieeexplore.iee.org.

Digital Object Identifier 10.1109/TWC.2016.2626291
Such antennas are strong candidates for $5 \mathrm{G}$ and beyond technologies where a single device may need to support multiple radio access technologies with different set of operational requirements (e.g., frequency band, polarization) [3]. In addition, the availability of multiple radiation patterns with different polarizations can provide enhanced spatial diversity that can fortify the techniques combating interference. One can imagine that each reconfigurable mode of operation of an MRA, a.k.a. antenna mode, creates a different antenna as it may have a different operation frequency, polarization and radiation pattern. Various enabling technologies (e.g., MEMS switching, semi-conductor switches, liquid metals) and design approaches (e.g., variable reactive loading, parasitic tuning and structural/material modifications) have been developed to create MRAs [1].

In this paper, we specifically consider the MRAs based on the parasitic tuning approach [4], [5]. In this MRA technology, an active antenna element is accompanied by a reconfigurable parasitic pixel layer whose pixels (electrically small rectangular shaped metallic elements) are interconnected by means of switching that are controlled via DC biased lines. By properly modifying the switch statuses, the parasitic surface layer is reshaped resulting in different radiation, frequency and polarization properties, i.e., a different antenna mode. We note, however, that the channel modelling as well as the analysis and design approaches developed throughout the paper can be applied to any MRA system, i.e., they are not specific to the parasitic tuning technology.

The use of MRAs in wireless communication systems has recently attracted significant attention due to the additional degrees of freedom they offer which may be exploited to achieve superior performance [6]-[12] as compared to systems employing antennas with fixed properties. Combined with the multiple-input multiple-output (MIMO) antenna technology, the resulting MR-MIMO antennas offer even greater degrees of freedom to combat the adverse effects of wireless propagation environments [13]. On the other hand, for such systems, extensive channel estimation requirements arise as a challenging and important issue. Since a rich scattering medium results in multipath propagation, changes in antenna radiation patterns result in different gains for different departure and arrival paths, and hence, their superposition results in different 
channels for each radiation pattern, giving rise to pattern diversity. Since each antenna mode creates a different channel, one needs to estimate the corresponding channel state information (CSI) separately. For cases with a few antenna modes, this overhead may be tolerable, however, if there exists a large number of antenna modes, the estimation overhead may be prohibitive. This is the main motivation of this work which attempts to develop efficient CSI estimation procedures for MR-MIMO systems.

Some attempts have been made to attack the channel estimation problem for MRAs in [14]-[16]. Eslami et al. [14] proposes a reduced complexity training approach that involves the selection of a number of modes and using only those modes for communication. The authors attempt to reduce the number of modes to be trained via statistical or direction finding based approaches where the effective angle of arrivals are determined to select better antenna modes. They also analyze the use of all available modes being trained for different pilot overheads. However, their approach does not allow for an effective utilization of the antenna modes dismissed from training sessions. In [15], Gulati and Dandekar propose a multi-bandit learning algorithm to select the antenna modes to reduce the required training overhead for CSI estimation. Again, the goal is to actively use a smaller number of antenna states for data transmission. In [16], Grau et. al. investigate the use of a class of generic reconfigurable antennas under a Kronecker channel correlation model and the assumption that a number of antenna ports can be decoupled. However, the decoupling assumption may not be valid for many MRA design approaches, and it may limit the available number of antenna modes for performance optimization.

Different from the previous approaches, in this paper, we first consolidate the relation among the antenna modes and the wireless channel. We formulate the relationship among the antenna modes associated with radiation fields from MR-MIMO antennas and the resulting channel realizations with the goal of developing low-overhead channel estimation techniques with reduced estimation errors. We consider a realistic 3D double-directional channel model for the MRMIMO antenna link and also assume that any of the available antenna modes can potentially be activated during transmission [17], [18]. Using the beamspace MIMO concept [19], the effects of MR-MIMO radiation field on the channel gains can be decoupled from those of the multipath propagation medium. This allows for casting the CSI estimation as an estimation/prediction problem where the antenna modes to be trained are used for obtaining a low-dimensional observation matrix from which the CSI for the other antenna modes are predicted. The selection of the training modes can further be optimized.

Our contributions can be summarized as follows: (i) an explicit relation among the antenna modes and the resulting channel gains are determined for MR-MIMO antennas, (ii) an efficient and effective channel estimation procedure is developed which utilizes a relatively small number of modes for explicit training and predicts the CSI for all available modes, and (iii) methods for antenna mode set selection to improve the CSI estimation performance are developed.
In our study, we consider the well-known Orthogonal Frequency Division Multiplexing (OFDM) based transmissions (e.g., as in the LTE and WiFi physical layers), and via extensive simulations, we show that about $25 \%$ theoretical throughput gain or about $3 \mathrm{~dB}$ signal-to-noise ratio (SNR) improvement can be achieved with respect to nonreconfigurable MIMO antenna systems in realistic scenarios. With suitable selection of training mode set according to the spatial correlation and the coherence time of the channel, we demonstrate that the proposed estimation/prediction technique can be employed to learn the CSI even when only a small set of antenna modes are explicitly trained.

The rest of the paper is organized as follows. In the next section, we provide the details on the underlying MRAs and MR-MIMO antennas, and extend an existing MIMO channel model to the case of MR-MIMO. In Section III, we describe the proposed channel estimation approaches for MR-MIMO systems. Extensive numerical examples are provided in Section IV, and finally, concluding remarks with some future research directions are given in Section V.

Notation: The notation $\vec{f}=f_{\theta} \vec{e}_{\theta}+f_{\phi} \vec{e}_{\phi}$ denotes spherical coordinate representation of complex electric field with $\vec{e}_{\theta}$ and $\vec{e}_{\phi}$ referring the unit vectors in $\theta$ and $\phi$ directions, respectively. $\langle\vec{f}, \vec{g}\rangle=f_{\theta}^{*} g_{\theta}+f_{\phi}^{*} g_{\phi}$ denotes the inner product where $(\cdot)^{*}$ indicates the complex conjugate operation. Similarly, the inner product for matrices, $\boldsymbol{C}_{N \times M}=$ $\left\langle\overrightarrow{\boldsymbol{A}}_{N \times L}, \overrightarrow{\boldsymbol{B}}_{L \times M}\right\rangle$ is defined as

$$
c_{n, m}=\sum_{l=1}^{L}\left\langle[\overrightarrow{\boldsymbol{A}}]_{n, l},[\overrightarrow{\boldsymbol{B}}]_{l, m}\right\rangle .
$$

$[\boldsymbol{A}]_{n, m}$ (or $[\overrightarrow{\boldsymbol{A}}]_{n, m}$ ) denotes the $n \times m^{\text {th }}$ entry of the matrix $\boldsymbol{A}$ (or $\left.\overrightarrow{\boldsymbol{A}}_{n, m}\right)$. $C N(a, b)$ denotes circularly symmetric complex Gaussian distribution with mean $a$ and variance $b . \boldsymbol{A} \otimes \boldsymbol{B}$ denotes the Kronecker product of $\boldsymbol{A}$ and $\boldsymbol{B}$, and $\operatorname{vec}(\boldsymbol{A})$ denotes vectorization of the matrix $\boldsymbol{A}$ by stacking its columns to a one dimensional vector. For a vector $\boldsymbol{v}, \operatorname{diag}(\boldsymbol{v})$ represents a diagonal matrix with elements of $\boldsymbol{v}$ on the main diagonal, and for a set of $L$ matrices $V_{i}, i=1, \ldots, L, \operatorname{diag}\left(V_{i}\right)$, $\forall i$ represents a block diagonal matrix constructed from $\boldsymbol{V}_{i}$ 's. $\boldsymbol{A}^{H}$ denotes the conjugate transpose of $\boldsymbol{A}$, and we use $\boldsymbol{A}^{-H}$ to indicate the inverse of $\boldsymbol{A}^{H}$.

\section{Channel Model for MR-MiMO Antenna Links}

The double directional MIMO (DD-MIMO) channel model [17], [20]-[23] is a widely accepted model that combines ideas from ray tracing and statistical channel modeling where a number of discrete direction of arrivals and departures are generated randomly using certain distributions. DD-MIMO channel model is suitable for accurately modelling the wireless channel taking into account the antenna radiation patterns. We first extend this model to the case of MR-MIMO antenna links, and then develop the corresponding signaling model.

\section{A. Legacy MIMO Versus MR-MIMO Antenna Links}

Fig. 1.a compares a legacy MIMO antenna having elements with fixed properties and an MR-MIMO antenna consisting of MRA elements (see Fig. 1.b) with variable properties. 


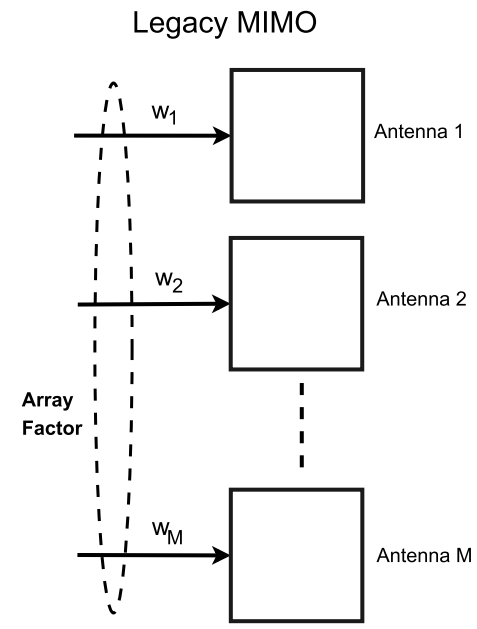

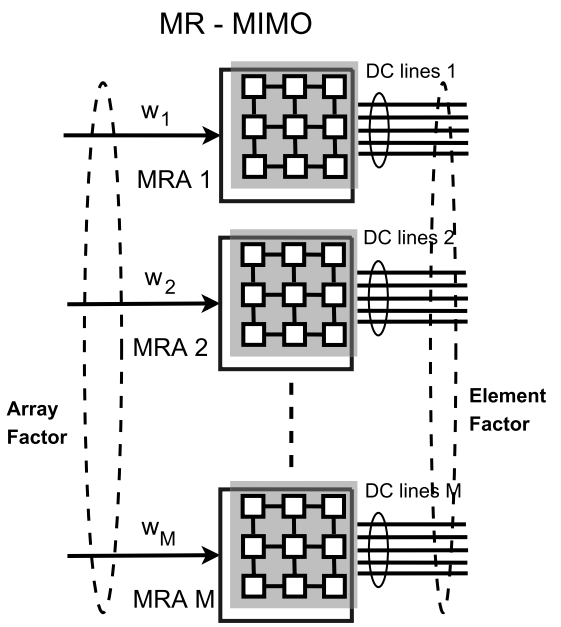

(a)

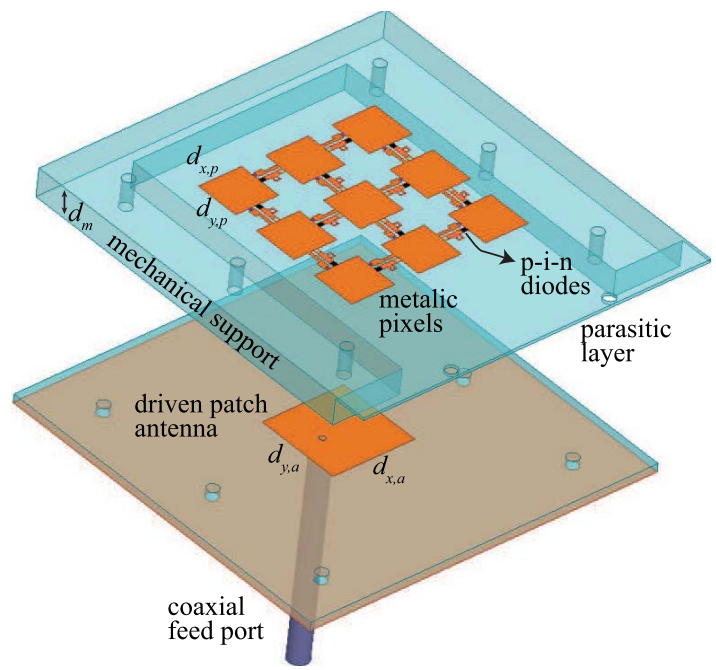

(b)

Fig. 1. (a) Legacy MIMO antenna versus MR-MIMO antenna, each with $M$ antenna elements. $w_{m}=\left|w_{m}\right| e^{j} L_{w_{m}}$ is the complex weight for antenna- $m$, $m=1, \ldots, M$. DC lines are used to excite the specific antenna modes $\mu_{m}, m=1, \ldots, M$, at antenna- $m$. (b) An MRA design based on parasitic coupling. A $3 \times 3$ metallic pixel surface interconnected by 12 on-off switches (e.g., via p-i-n diodes).

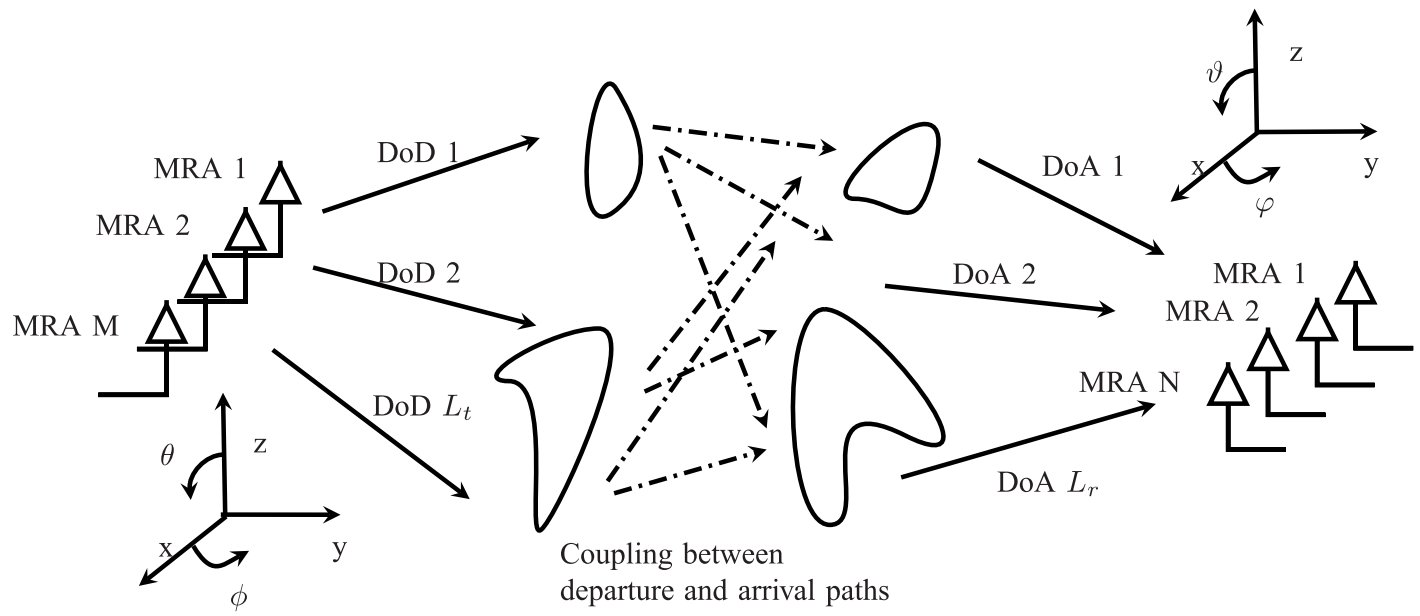

Fig. 2. Double directional channel model. $M$ transmit and $N$ receive MRA elements, $L_{t}$ direction of departures, and $L_{r}$ direction of arrivals. The reference coordinates are also depicted for the transmit and receive antennas.

MRA elements provide additional degrees of freedom to the MIMO system due to the variable element factors. For the MRA design depicted in Fig. 1.b, this is accomplished by changing the geometry of the parasitic surface via the on-off switches embedded in between the metallic pixels constituting the parasitic layer. Due to space constraints, we refer the reader to [24] for details on the design and optimization of this MRA design approach. The MRA depicted in Fig. 1.b comprises of a $3 \times 3$ parasitic pixel surface interconnected by $12 \mathrm{p}-\mathrm{i}-\mathrm{n}$ diode switches. Thus, a total of $2^{12}$ different switch states, i.e., antenna modes, exist. A given set of switch states define a specific polarization, frequency and radiation pattern, which is referred to as the mode of operation.

In this paper, we focus on MR-MIMO antennas with identical reconfigurable elements, but each element may be set to a different mode. Let $\vec{f}(\theta, \phi, \mu)$ denote the elemental complex far-field radiation pattern where $\mu \in\left\{1,2, \ldots, L_{\mu}\right\}$ is the antenna mode index representing the excited mode of operation and $L_{\mu}$ is the number of antenna modes. Then, for an MR-MIMO antenna with $M$ MRA elements, ignoring the mutual coupling among the parasitic surfaces of different elements, the complex pattern for the $m^{\text {th }}$ element can be expressed as

$$
\vec{f}\left(\theta, \phi, \mu_{m}\right)=f_{\theta}\left(\theta, \phi, \mu_{m}\right) \vec{e}_{\theta}+f_{\phi}\left(\theta, \phi, \mu_{m}\right) \vec{e}_{\phi}
$$

with $\mu_{m} \in\left\{1, \ldots, L_{\mu}\right\}$ representing the mode index of antenna- $m, m=1, \ldots, M$. Note that there are $L_{\mu}^{M}$ different modes of operation corresponding to different radiation patterns.

\section{B. Double Directional MIMO Channel Model for MR-MIMO Systems}

Fig. 2 illustrates the double directional channel model [20], [21] for an MR-MIMO system with $M$ transmit and $N$ receive MRAs. According to this model, under the balanced array [20] and plane wave propagation assumptions, 
TABLE I

Double Directional Mimo Model Parameters

\begin{tabular}{|c|c|c|c|}
\hline Parameter & Definition & Parameter & Definition \\
\hline$\overline{L_{t}}$ & Number of departure paths & $L_{r}$ & Number of arrival paths \\
\hline $\overrightarrow{\boldsymbol{k}}(\theta, \phi)$ & Unit length wave-vector for departure paths & $\overrightarrow{\boldsymbol{k}}(\vartheta, \varphi)$ & Unit length wave-vector for arrival paths \\
\hline$\left(\theta_{i}, \phi_{i}\right)$ & Angle of departure for DoD- $i, i=1, \ldots, L_{t}$ & $\left(\vartheta_{j}, \varphi_{j}\right)$ & Angle of arrival for DoA- $j, j=1, \ldots, L_{r}$ \\
\hline$\left(\theta_{B}, \phi_{B}\right)$ & Transmit antenna tilt direction & $\left(\vartheta_{B}, \varphi_{B}\right)$ & Receive antenna tilt direction \\
\hline$x_{j, i}$ & Channel gain for the path between DoD- $i$ and DoA- $j$ & $\varsigma_{d, j}$ & $\begin{array}{l}\text { Doppler shift for } j^{\text {th }} \text { arrival path, } \varsigma_{d, j}= \\
\frac{f_{c}|\boldsymbol{v}|}{c} \boldsymbol{k}^{T}\left(\vartheta_{j}, \varphi_{j}\right) \boldsymbol{v}, \boldsymbol{v} \text { : Velocity vector }\end{array}$ \\
\hline$\tau_{j, i}$ & Delay for the path between DoD- $i$ and DoA- $j$ & $\lambda$ & wavelength \\
\hline $\boldsymbol{p}_{m}$ & Transmit antenna- $m$ coordinate & $\boldsymbol{q}_{n}$ & Receive antenna- $n$ coordinate \\
\hline$\mu$ & $\begin{array}{l}M \text { transmit antenna mode indices }\left(\mu_{1}, \ldots, \mu_{M}\right) \text {, } \\
\mu \in\left\{1, \ldots, L_{\mu}\right\}, m=1, \ldots, M\end{array}$ & $\nu$ & $\begin{array}{l}N \text { receive mode indices }\left(\nu_{1}, \ldots, \nu_{N}\right), \nu_{n} \in \\
\left\{1, \ldots, L_{\nu}\right\}, n=1, \ldots, N\end{array}$ \\
\hline$L_{\mu}$ & Number of modes for transmit MRA element & $L_{\nu}$ & Number of modes for receive MRA element \\
\hline $\overrightarrow{f_{\Omega}}\left(\theta, \phi, \mu_{m}\right)$ & $\begin{array}{l}\text { E-field pattern for the } m^{\text {th }} \text { transmit MRA ele- } \\
\text { ment taking into account the boresight tilt direction } \\
\left(\theta_{B}, \phi_{B}\right)\end{array}$ & $\overrightarrow{f_{\Psi}}\left(\vartheta, \varphi, \nu_{n}\right)$ & $\begin{array}{l}\text { E-field pattern for the } n^{\text {th }} \text { receive MRA element } \\
\text { taking into account the boresight tilt direction } \\
\left(\vartheta_{B}, \varphi_{B}\right)\end{array}$ \\
\hline
\end{tabular}

and assuming that the channel is fixed during the symbol duration, the complex base-band channel gain between transmit MRA- $m$ and receive MRA- $n$ for a narrow-band signal at frequency $\varsigma$ can be expressed as

$$
\begin{aligned}
h_{n, m} & \left(\mu_{m}, v_{n}\right) \\
= & \sum_{j=1}^{L_{r}} \sum_{i=1}^{L_{t}} x_{j, i}\left\langle\vec{f}_{\Omega}\left(\theta_{i}, \phi_{i}, \mu_{m}\right), \vec{f}_{\Psi}\left(\vartheta_{j}, \varphi_{j}, v_{n}\right)\right\rangle \\
\quad & \times e^{-j \frac{2 \pi}{\lambda}\left(\boldsymbol{k}^{T}\left(\theta_{i}, \phi_{i}\right)\left(\boldsymbol{p}_{m}-\boldsymbol{p}_{1}\right)+\boldsymbol{k}^{T}\left(\vartheta_{j}, \varphi_{j}\right)\left(\boldsymbol{q}_{n}-\boldsymbol{q}_{1}\right)\right)-j 2 \pi \varsigma \tau_{i, j}}
\end{aligned}
$$

where the parameters are summarized in Table I.

Let $[\boldsymbol{H}]_{n, m}(\boldsymbol{\mu}, \boldsymbol{v})=h_{n, m}\left(\mu_{m}, v_{n}\right)$ denote the $N \times M$ MIMO channel matrix. Each departure path is coupled with each of the arrival paths resulting in a total of $L_{t} L_{r}$ resolvable paths whose gain and propagation delay are denoted by $x_{j, i}$ and $\tau_{j, i}$, respectively, $i=1, \ldots, L_{t}, j=1, \ldots, L_{r}$. We denote the $L_{r} \times L_{t}$ channel gain matrix by $\boldsymbol{X}$.

To express (2) in a more compact form, let us first define the transmit and receive steering matrices for the transmit and arrival paths as, $\boldsymbol{B}=\left[\begin{array}{lll}\boldsymbol{b}_{1} & \ldots & \boldsymbol{b}_{L_{t}}\end{array}\right]$ and $\boldsymbol{A}=\left[\begin{array}{lll}\boldsymbol{a}_{1} & \ldots & \boldsymbol{a}_{L_{r}}\end{array}\right]$, respectively, where $\boldsymbol{b}_{i}$ and $\boldsymbol{a}_{j}$ are the transmit and receive steering vectors given by

$$
\begin{aligned}
& \boldsymbol{b}_{i}=\left[1 e^{-j \frac{2 \pi}{\lambda} \boldsymbol{k}^{T}\left(\theta_{i}, \phi_{i}\right)\left(\boldsymbol{p}_{2}-\boldsymbol{p}_{1}\right)} \ldots e^{-j \frac{2 \pi}{\lambda} \boldsymbol{k}^{T}\left(\theta_{i}, \phi_{i}\right)\left(\boldsymbol{p}_{M}-\boldsymbol{p}_{1}\right)}\right]^{T} \\
& \text { for } i=1, \ldots, L_{t}, \text { and } \\
& \boldsymbol{a}_{j}=\left[1 e^{-j \frac{2 \pi}{\lambda} \boldsymbol{k}^{T}\left(\vartheta_{j}, \varphi_{j}\right)\left(\boldsymbol{q}_{2}-\boldsymbol{q}_{1}\right)} \ldots e^{-j \frac{2 \pi}{\lambda} \boldsymbol{k}^{T}\left(\vartheta_{j}, \varphi_{j}\right)\left(\boldsymbol{q}_{N}-\boldsymbol{q}_{1}\right)}\right]^{T},
\end{aligned}
$$

for $j=1, \ldots, L_{r}$. Next, by defining the pattern vectors

$$
\begin{aligned}
\vec{f}_{\Omega}\left(\mu_{m}\right) & =\left[\vec{f}_{\Omega}\left(\theta_{1}, \phi_{1}, \mu_{m}\right), \ldots, \vec{f}_{\Omega}\left(\theta_{L_{t}}, \phi_{L_{t}}, \mu_{m}\right]^{T}\right. \\
\vec{f}_{\Psi}\left(v_{n}\right) & =\left[\vec{f}_{\Psi}\left(\vartheta_{1}, \varphi_{1}, v_{n}\right), \ldots, \vec{f}_{\Psi}\left(\vartheta_{L_{r}}, \varphi_{L_{r}}, v_{n}\right)\right]^{T}
\end{aligned}
$$

and expressing the rows of the steering matrices as diagonal matrices

$\boldsymbol{U}_{m}=\operatorname{diag}\left(\left[b_{m, 1} \ldots b_{m, L_{t}}\right]\right), b_{m, i}=[\boldsymbol{B}]_{m, i}, i=1, \ldots, L_{t}$,

$\boldsymbol{V}_{n}=\operatorname{diag}\left(\left[a_{n, 1} \ldots a_{n, L_{r}}\right]\right), a_{n, i}=[\boldsymbol{A}]_{n, j}, j=1, \ldots, L_{r}$, one can rewrite (2) as

$$
\begin{aligned}
h_{n, m}\left(\mu_{m}, v_{n}\right)= & \boldsymbol{f}_{\Omega, \theta}^{H}\left(\mu_{m}\right) \boldsymbol{U}_{m} \boldsymbol{X}^{\prime T} \boldsymbol{V}_{n} \boldsymbol{f}_{\Psi, \theta}\left(v_{n}\right) \\
& +\boldsymbol{f}_{\Omega, \phi}^{H}\left(\mu_{m}\right) \boldsymbol{U}_{m} \boldsymbol{X}^{\prime T} \boldsymbol{V}_{n} \boldsymbol{f}_{\Psi, \phi}\left(v_{n}\right) \\
= & \left\langle\overrightarrow{\boldsymbol{f}}_{\Omega}\left(\mu_{m}\right), \tilde{\boldsymbol{H}}_{n, m} \overrightarrow{\boldsymbol{f}}_{\Psi}\left(v_{n}\right)\right\rangle
\end{aligned}
$$

where $\left[\boldsymbol{X}^{\prime}\right]_{j, i}=x_{j, i} e^{j 2 \pi \varsigma_{d, j} t} e^{-j 2 \pi \varsigma \tau_{j, i}}$, and

$\left[\tilde{\boldsymbol{H}}_{n, m}\right]_{i, j}=[\boldsymbol{B}]_{m, i} \cdot[\boldsymbol{A}]_{n, j} \cdot\left[\boldsymbol{X}^{\prime}\right]_{j, i}, i=1, \ldots, L_{t}, j=1, \ldots, L_{r}$.

Note that (5) decouples the impact of element-wise reconfiguration of the MR-MIMO antenna from the other terms related to steering vectors and the multipath propagation effects. In addition, the inner product expression among the electric field vectors reflects the impact of the field polarization mismatch among the received signal polarization and antenna polarizations [25], [26]. Furthermore, (5) shows explicitly the dependence of the channel variations on the scatterers and the user speed through $x_{j, i}$ and $\varsigma_{d, j}$. In what follows, it is assumed that the scatterers are quasistatic, that is, they remain the same over a long period of time, while the temporal variations due to $\varsigma_{d, j}$ are more pronounced. Note that for MR-MIMO antennas, (5) can be employed to generate the channel matrix $\boldsymbol{H}_{n, m}(\boldsymbol{\mu}, \boldsymbol{v})$. For legacy MIMO with identical elements, this relation can be simplified to $\boldsymbol{H}=\boldsymbol{B} \boldsymbol{H}_{s} \boldsymbol{A}^{T}$ where $\boldsymbol{H}_{s}$ is the $L_{t} \times L_{r}$ matrix whose entries are the complex path gains between all departure and arrival angles including the associated antenna gains [20].

Using the superposition principle [25], [26], after matched filtering [27, Ch. 4], the signal received from antenna- $n$ for a narrow-band transmission can be written as

$$
r_{n}\left(\boldsymbol{\mu}, v_{n}\right)=\sum_{m=1}^{M} h_{n, m}\left(\mu_{m}, v_{n}\right) w_{m} s_{m}+z_{n}
$$

where it is assumed that the channel is fixed during the symbol transmission time, and $z_{n} \sim C N\left(0, \sigma_{z}^{2}\right)$ denotes the additive white Gaussian noise at the receive antenna- $n$. In (6), $s_{m}$ denotes the unit power signal emanating from antenna- $m$, and $w_{m}$ is the complex gain weighting at that antenna. From (5) and (6), it is seen that changing the transmit and/or receive antenna modes result in different channel realizations for 
the same propagation medium. This point will be elaborated further in the following sections.

\section{Channel Estimation FOR MR-MiMO ANTENNA SYSTEMS}

Employing MRAs at the transmitter and/or receiver introduces variations due to antenna reconfigurations in addition to the usual temporal and spatial variations created by multipath fading. Each one of the large number of antenna modes associated with an MRA creates a different channel, thereby making channel estimation a challenging task. In order to fully exploit the degrees of freedom available, it is necessary to estimate the channel for different (perhaps all) antenna modes within the coherence time of the channel. With the presence of a large number of modes (e.g., 4096 for the MRA shown in Fig. 1.b), the channel estimation based on an exhaustive training requires excessive overhead and delay, which makes it undesirable, or even infeasible. Therefore, an efficient MIMO channel estimation procedure for MRA systems requires a unified framework taking into account both the structure of the wireless medium and the underlying MRA radiation capabilities. With this motivation, we utilize the beamspace representation of radiation patterns [28]-[30] and resulting wireless channels [31]-[33], and develop an analytical framework for the combined estimation and prediction procedure. The explicit relation in (5) among the underlying antenna modes and the channel realizations enables the development of a formulation where only a small set of antenna modes need to be explicitly trained, and the remaining modes are predicted using the correlations among them.

\section{A. Antenna Patterns and the Wireless Channel}

Equation (5) relates the underlying antenna radiation patterns and the resulting channel realizations. This relation, however, requires the explicit knowledge of departure and arrival angles. To avoid this issue and evaluate the impact of different radiation patterns on the CSI, one can utilize the beamspace concept of [19, Ch. 3] and [21]. To that end, let there exist $L_{\mu}$ antenna modes for the element of an MR-MIMO antenna, and therefore, $L_{\mu}$ different radiation patterns, $\vec{f}(\theta, \phi, \mu)$, $\mu \in\left\{1, \ldots, L_{\mu}\right\}$. In addition, let there be $\digamma \leq L_{\mu}$ orthonormal basis radiation pattern functions, $\vec{\omega}_{i}(\theta, \phi)$, $i=1, \ldots, \digamma$, which can be used to represent all the radiation patterns. For instance, for a given set of radiation pattern functions $\vec{f}(\theta, \phi, \mu), \mu \in\left\{1, \ldots, L_{\mu}\right\}$, the Gram-Schmidt process can be used to compute one such basis function set [19, Ch. 3]. Thus, we have the analysis-synthesis relations as

$$
\begin{aligned}
\vec{f}(\theta, \phi, \mu) & =\sum_{i=1}^{\digamma} \alpha_{\mu, i} \vec{\omega}_{i}(\theta, \phi) \\
\text { s.t. } \alpha_{\mu, i} & =\iint\left\langle\vec{f}(\theta, \phi, \mu), \vec{\omega}_{i}(\theta, \phi)\right\rangle \sin (\theta) d \theta d \phi .
\end{aligned}
$$

From (7), we can express correlation among radiation patterns corresponding to modes $\mu_{k}$ and $\mu_{l}$ as

$$
\breve{\rho}_{\mu_{k}, \mu_{l}}=\frac{\boldsymbol{\alpha}_{\mu_{k}}^{H} \boldsymbol{\alpha}_{\mu_{l}}}{\left\|\boldsymbol{\alpha}_{\mu_{k}}\right\| \cdot\left\|\boldsymbol{\alpha}_{\mu_{l}}\right\|}
$$

where $\boldsymbol{\alpha}_{\mu}=\left[\alpha_{\mu, 1} \ldots \alpha_{\mu, \digamma}\right]$. Applying (7) to both the transmit and receive radiation patterns in (5) (for brevity, we drop the $(t, f)$ for this section), we obtain

$$
\begin{aligned}
h_{n, m}\left(\mu_{m}, v_{n}\right) & =\left\langle\overrightarrow{\boldsymbol{\Omega}} \boldsymbol{\alpha}_{\mu_{m}}, \tilde{\boldsymbol{H}}_{n, m} \overrightarrow{\boldsymbol{\Psi}} \boldsymbol{\gamma}_{v_{n}}\right\rangle \\
& =\boldsymbol{\alpha}_{\mu_{m}}^{H}\left\langle\overrightarrow{\boldsymbol{\Omega}}, \tilde{\boldsymbol{H}}_{n, m} \overrightarrow{\boldsymbol{\Psi}}\right\rangle \boldsymbol{\gamma}_{\nu_{n}} \\
& =\boldsymbol{\alpha}_{\mu_{m}}^{H} \overline{\boldsymbol{H}}_{n, m} \boldsymbol{\gamma}_{\boldsymbol{v}_{m}} \\
& =\left(\boldsymbol{\gamma}_{v_{n}}^{*} \otimes \boldsymbol{\alpha}_{\mu_{m}}\right)^{H} \operatorname{vec}\left(\overline{\boldsymbol{H}}_{n, m}\right)
\end{aligned}
$$

where $\boldsymbol{\alpha}_{\mu_{m}}$ and $\boldsymbol{\gamma}_{v_{n}}$ are, respectively, the $\digamma_{t} \times 1$ and $\digamma_{r} \times 1$ synthesis coefficients for transmit and receive patterns, $\overrightarrow{\boldsymbol{\Omega}}$ is the $L_{t} \times \digamma_{t}$ basis radiation pattern values evaluated at $\left(\theta_{i}, \phi_{i}\right)$, for $i=1, \ldots, L_{t}$, and $\overrightarrow{\boldsymbol{\Psi}}$ is the $L_{r} \times \digamma_{r}$ basis radiation pattern values evaluated at $\left(\vartheta_{i}, \varphi_{i}\right)$, for $i=1, \ldots, L_{r}$. To obtain (9), we collect the terms other than the antenna mode configuration into an $\digamma_{t} \times \digamma_{r}$ matrix using $\overline{\boldsymbol{H}}_{n, m}=\left\langle\overrightarrow{\boldsymbol{\Omega}}, \tilde{\boldsymbol{H}}_{n, m} \overrightarrow{\boldsymbol{\Psi}}\right\rangle$. Hence, all the unknown variables appear in the matrix $\overline{\boldsymbol{H}}_{n, m}$. With the exact knowledge of this matrix, along with the synthesis coefficients $\boldsymbol{\alpha}_{\mu_{m}}$ and $\boldsymbol{\gamma}_{v_{n}}$ that can be calculated off-line, (9) can be used to evaluate the relation among the element radiation patterns and the channel gains for all the antenna modes.

Using (9), the cross correlation between the channel with modes $\mu_{m}=i, v_{n}=j$ and $\mu_{m}=k, v_{n}=l$, denoted by $\rho_{n, m}(i, j, k, l)$, is given by

$$
\begin{aligned}
& \rho_{n, m}(i, j, k, l) \\
& =\frac{E\left\{h_{n, m}(i, j) h_{n, m}^{*}(k, l)\right\}}{\sqrt{E\left\{\left\|h_{n, m}(i, j)\right\|^{2}\right\} E\left\{\left\|h_{n, m}(k, l)\right\|^{2}\right\}}} \\
& =\frac{\operatorname{Tr}\left(\left(\boldsymbol{\gamma}_{l}^{*} \boldsymbol{\gamma}_{j}^{T} \otimes \boldsymbol{\alpha}_{k} \boldsymbol{\alpha}_{i}^{H}\right) \boldsymbol{R}_{\bar{x}}\right)}{\sqrt{\operatorname{Tr}\left(\left(\boldsymbol{\gamma}_{j}^{*} \boldsymbol{\gamma}_{j}^{T} \otimes \boldsymbol{\alpha}_{i} \boldsymbol{\alpha}_{i}^{H}\right) \boldsymbol{R}_{\bar{x}}\right) \operatorname{Tr}\left(\left(\boldsymbol{\gamma}_{l}^{*} \boldsymbol{\gamma}_{l}^{T} \otimes \boldsymbol{\alpha}_{k} \boldsymbol{\alpha}_{k}^{H}\right) \boldsymbol{R}_{\bar{x}}\right)}}
\end{aligned}
$$

where $\overline{\boldsymbol{x}}=\operatorname{vec}\left(\overline{\boldsymbol{H}}_{n, m}\right), \boldsymbol{R}_{\overline{\boldsymbol{x}}}=E\left\{\overline{\boldsymbol{x}} \overline{\boldsymbol{x}}^{H}\right\}$, and we exploit the relation $\operatorname{Tr}(\boldsymbol{A} \boldsymbol{B})=\operatorname{Tr}(\boldsymbol{B} \boldsymbol{A})$. It is seen that as long as the correlation matrix $\boldsymbol{R}_{\bar{x}}$ remains same, the correlation between the channels for the different modes of operation remains the same. This observation is important especially for a slowly varying channel where the scatterers are quasistatic and channel realization for a given mode can be predicted from the channel realization of another or some other modes using the correlations $\rho_{n, m}(\cdot)$. The channel correlations between different modes of operation can also be justified directly from (2) where it is observed that a small change in radiation pattern functions can create only a small change in respective channel realizations. The changes in the channel gain depend on the similarities among the antenna radiation patterns. Equation (10) provides a mathematical framework for this observation.

The relation in (10) can further be simplified provided that the medium is a uniformly rich scattering environment where $\boldsymbol{R}_{\bar{x}}$ is modelled as an identity matrix, i.e.,

$$
\rho_{n, m}(i, j, k, l)=\frac{\left(\boldsymbol{\gamma}_{j}^{T} \boldsymbol{\gamma}_{l}^{*}\right) \cdot\left(\boldsymbol{\alpha}_{i}^{H} \boldsymbol{\alpha}_{k}\right)}{\left\|\boldsymbol{\gamma}_{j}\right\| \cdot\left\|\boldsymbol{\gamma}_{l}\right\| \cdot\left\|\boldsymbol{\alpha}_{i}\right\| \cdot\left\|\boldsymbol{\alpha}_{k}\right\|}
$$

which depicts a more clear relation between the pattern and channel correlations. That is, those antenna modes that have 


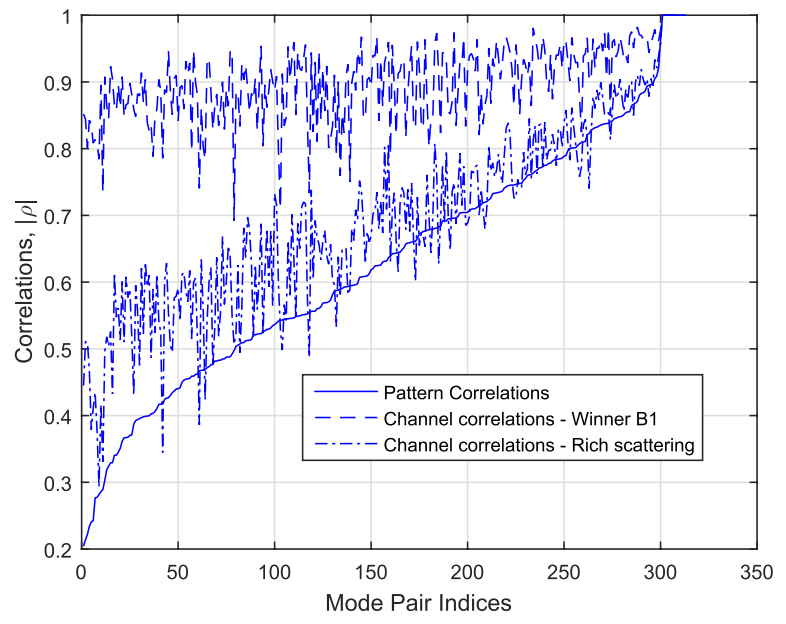

Fig. 3. Pattern correlations versus channel correlations. The channels are averaged over 20 different multipath conditions each with 10000 channel realizations.

smaller pattern cross-correlations will result in smaller channel correlations. If the mode of the transmit or the receive antenna is fixed, one can further simplify (11) to

$$
\begin{aligned}
\rho_{n, m}\left(i_{0}, j, i_{0}, l\right) & =\frac{\boldsymbol{\gamma}_{j}^{T} \boldsymbol{\gamma}_{l}^{*}}{\left\|\boldsymbol{\gamma}_{j}\right\| \cdot\left\|\boldsymbol{\gamma}_{l}\right\|}, \\
\rho_{n, m}\left(i, j_{0}, k, j_{0}\right) & =\frac{\boldsymbol{\alpha}_{i}^{H} \boldsymbol{\alpha}_{k}}{\left\|\boldsymbol{\alpha}_{i}\right\| \cdot\left\|\boldsymbol{\alpha}_{k}\right\|},
\end{aligned}
$$

where $i_{0}$ denotes the transmit MRA mode, and $j_{0}$ denotes the receive MRA mode indices in (12) and (13), respectively. For (11)-(13), it is seen that the channel correlations reduce to radiation pattern correlations defined in (8). We note that the expressions in (11)-(13) are provided to highlight the relationship between the pattern and channel correlations. For actual system analysis and numerical examples in the subsequent sections, we employ (10) to evaluate the channel correlations.

Let us study the relationship between different antenna modes and the resulting channel gains using a numerical example. Fig. 3 depicts the relations among the pattern correlations using (10) and the corresponding realized channel correlations obtained by changing the antenna mode while keeping the propagation medium fixed. We investigate both a relatively low scattering environment modeled by micro-cell channel model B1 from Winner+ project [34]-[37], and a uniformly rich scattering environment modeled via 400 transmit and 400 receive rays with $3 \mathrm{D}$ uniform AoD and AoA spreads. In this example, the receive antenna mode is fixed and the channels are generated for 25 different transmit antenna modes, which amounts to a total of 325 auto- and cross-correlation values. The correlation values are sorted according to the absolute value of the radiation pattern correlations, and at each index, the corresponding channel correlation is plotted. In both cases, it is seen that as the radiation pattern correlations increase, the channel correlations tend to increase as well; however, this relationship is more pronounced for a uniform scattering environment. This result clearly demonstrates that the CSI estimates for a number of modes will help predict the CSI for the others.

\section{B. Channel Estimation for MR-MIMO Links}

We consider pilot-assisted training with narrow-band transmission where a known pilot signal sequence is intermittently transmitted to allow the receiver to perform channel estimation. In addition, we assume an orthogonal training mechanism for the MIMO case where only one transmit antenna is active for a given training symbol duration.

Let $T_{c}$ and $K_{c}$ denote the number of symbols and subcarriers, respectively, in a channel training and data transmission session, and let $\left(t_{i}, k_{i, j}\right), i=1, \ldots, L_{p}, j=1, \ldots, K_{i}$, denote the symbol and subcarrier indices of the resource elements (REs) at which pilot signals, $p_{m}, m=1, \ldots, M$, from antenna- $m$ are transmitted. Assuming a symbol duration $T_{s}$, we have $t=t_{i} T_{s}$, and $\varsigma=k_{i, j} \Delta f . L_{p}$ is the number of training symbols (e.g., channel uses) such that $1 \leq t_{1}<$ $\ldots<t_{L_{p}} \leq T_{c}$, and $K_{i}$ is the number of training subcarriers for symbol- $i$ such that $1 \leq K_{i} \leq K_{c}$. Hence, a total of $L_{T}=\sum_{i=1}^{L_{p}} K_{i}$ out of $T_{c} K_{c}$ subcarriers are employed for training. The received signal at antenna- $n, n=1, \ldots, N$, is given by

$$
r_{n}\left(\boldsymbol{\mu}_{t_{i}}, v_{n, t_{i}}\right)=\sum_{m=1}^{M} h_{n, m}\left(\mu_{m, t_{i}}, v_{n, t_{i}}\right) p_{m, t_{i}, k_{i, j}}+z_{n, t_{i}, k_{i, j}},
$$

for $i=1, \ldots, L_{p}, j=1, \ldots, K_{i}$, which can be rewritten as

$$
\boldsymbol{r}(\mu, v)=\boldsymbol{P h}(\mu, v)+z
$$

by stacking the $r_{n}\left(\boldsymbol{\mu}_{t_{i}}, v_{n, t_{i}}\right)$ and $z_{n, t_{i}, k_{i, j}}$ to $N L_{T} \times 1$ vectors, $h_{n, m}\left(\mu_{m, t_{i}}, v_{n, t_{i}}\right)$ to $M N L_{T} \times 1$, vector and expressing the pilot sequence as a block diagonal matrix $\boldsymbol{P}=\operatorname{diag}\left\{\boldsymbol{p}_{i, j}^{T} \otimes\right.$ $\left.\boldsymbol{I}_{N \times N}\right\}, i=1, \ldots, L_{p}, j=1, \ldots, K_{i}$, with $\boldsymbol{p}_{i, j}=$ $\left[p_{1, t_{i}, k_{i, j}} \ldots p_{M, t_{i}, k_{i, j}}\right]^{T}$. The minimum mean square error estimation (MMSE) [38] for the channel and the corresponding mean square error covariance matrix are then given by

$$
\begin{aligned}
\hat{\boldsymbol{h}}(\boldsymbol{\mu}, \boldsymbol{v}) & =\boldsymbol{R}_{\boldsymbol{h} \boldsymbol{h}}\left(\boldsymbol{R}_{\boldsymbol{h} \boldsymbol{h}}+\boldsymbol{P}^{-1} \boldsymbol{R}_{z z} \boldsymbol{P}^{-H}\right)^{-1} \boldsymbol{P}^{-1} \boldsymbol{r}(\boldsymbol{\mu}, \boldsymbol{v}), \\
\boldsymbol{R}_{\boldsymbol{\epsilon} \boldsymbol{\epsilon}}(\boldsymbol{\mu}, \boldsymbol{v}) & =\boldsymbol{R}_{\boldsymbol{h} \boldsymbol{h}}-\boldsymbol{R}_{\boldsymbol{h} \boldsymbol{h}}\left(\boldsymbol{R}_{\boldsymbol{h} \boldsymbol{h}}+\boldsymbol{P}^{-1} \boldsymbol{R}_{z z} \boldsymbol{P}^{-H}\right)^{-1} \boldsymbol{R}_{\boldsymbol{h} \boldsymbol{h}},
\end{aligned}
$$

respectively. Here $\boldsymbol{R}_{\boldsymbol{h} \boldsymbol{h}}$ and $\boldsymbol{R}_{z z}=\sigma_{z}^{2} I_{M N L_{T}}$ are the covariance matrices of the channel vector and the noise vector, respectively. Note that $\boldsymbol{h}$ is a vector containing channel gains at REs at which pilot symbols are transmitted over different antennas, and hence $\boldsymbol{R}_{\boldsymbol{h} \boldsymbol{h}}$ contains temporal, spectral and spatial correlations.

Note that for reduced training overhead, the amount of training symbols should be kept small. Typically, a portion of the subcarriers are reserved for pilot transmission in an OFDM symbol which means that data is also being transmitted concurrently. Let us assume that the mode training procedure is activated from time to time. That is, during a regular transmission period, the transmitter uses the selected antenna mode for both training and data transmission. During the mode training session, the transmitter activates $F$ antenna modes meanwhile usual data transmission is performed at the data subcarriers. During the training period, ideas similar to adaptive modulation and coding [39], [40] can be used to take into account the excitation of different antenna modes. Finally, we note that we consider the transmit antenna reconfiguration 


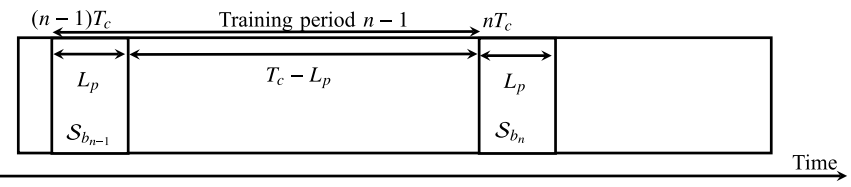

Fig. 4. Channel training protocol for transmission with reconfigurable antennas. Each training period of $T_{c}$ channel uses employs $L_{p}$ channel uses for training the mode group $S_{b_{n}}$ for the $n^{\text {th }}$ training session, with $b_{n}=\left(n \bmod \frac{L_{\mu}}{F}\right)$. With fixed mode groups, $S_{b}, b=0, \ldots, \frac{L_{\mu}}{F}-1$, this protocol corresponds to exhaustive mode training. The training groups can ba updated according to intelligent mode update criteria as well.

while keeping receive antenna at a fixed configuration in the following. It is straightforward to extend the proposed concepts to the general case where both transmitter and receiver perform mode configuration.

\section{Antenna Mode Training for MR-MIMO}

When there are many antenna modes, it may not be feasible to train all the modes during one coherence block. To develop a complete scheme, let us first extend the periodic-training based channel estimation procedure to a MR-MIMO link where the channels for a relatively small number of antenna modes are to be estimated.

1) Exhaustive Training: Out of the all possible $L_{\mu}$ transmit modes, the transmitter and receiver arbitrarily agree on the set of transmit antenna mode sets

$$
S_{b}=\left\{\mu_{b, 1}, \ldots, \mu_{b, F}\right\}
$$

for the $b^{\text {th }}$ antenna mode group. There are a total of $L_{\mu} / F$ different groups to be employed for transmission. Each antenna mode is trained for an equal number of channel uses denoted by $\beta=\frac{L_{p}}{F}$. As depicted in Fig. 4 , the channel training with the subsequent mode group starts $T_{c}$ channel uses later. It is assumed that the training groups are repeated according to modulo- $L_{\mu} / F$ if the total transmission time to the user takes longer than $\frac{L_{\mu} T_{c}}{F}$ channel uses. During the initial $L_{p}$ channel uses of the training period, the receiver estimates the channel for each mode and reports the best modes to the transmitter. The transmitter uses the selected mode for the rest of the coherence block until the next channel training period. Note that with this approach, regardless of the number of antenna modes, the training overhead is fixed at $L_{T} / T_{c} K_{c}$. Reducing the number of training symbols per antenna mode results in larger delays for CSI estimation of the remaining modes. Later, it will be shown by numerical examples that the selection of the design parameters strongly depends on the channel coherence time and the correlations among different antenna modes.

2) Intelligent Mode Update: While small scale fading causes relatively fast temporal variations, the higher order variations, such as spatial correlation of the MIMO channel, vary much more slowly. This is mainly due to the fact that the main scatterers do not change significantly for low-mobility users over multiple coherence blocks [20]. This implies that certain antenna modes will better fit to a propagation environment. Thus, as transmissions go on, the system may be able to learn the appropriate antenna modes and limit the antenna reconfiguration to those.

To that end, we assume that there is a low-rate feedback channel where the antenna modes selected by the receiver are fed back to the transmitter. The transmitter and receiver initially agree on the set of antenna mode pairs as in the exhaustive mode training approach. The receiver then monitors the system performance during data transmission periods and updates the training mode groups $S_{b}$ according to some criterion. For example, for a training mode pair $(\boldsymbol{\mu}, \boldsymbol{v})$, assuming equal power allocation, one such metric is the average achievable rate estimate

$$
C(\boldsymbol{\mu}, \boldsymbol{v})=\frac{1}{U_{\boldsymbol{\mu}, \boldsymbol{v}}} \sum_{i, j, \boldsymbol{\mu}_{t_{i}}=\boldsymbol{\mu}, \boldsymbol{v}_{t_{i}}=\boldsymbol{v}} \log _{2}\left|\boldsymbol{I}+\frac{1}{\sigma_{z}^{2}} \hat{\boldsymbol{H}}(\boldsymbol{\mu}, \boldsymbol{v}) \hat{\boldsymbol{H}}^{H}(\boldsymbol{\mu}, \boldsymbol{v})\right|
$$

where $U_{\boldsymbol{\mu}, \boldsymbol{v}}$ is the number of REs for which the mode pair $(\boldsymbol{\mu}, \boldsymbol{v})$ is trained, and $[\hat{\boldsymbol{H}}(\boldsymbol{\mu}, \boldsymbol{v})]_{n, m}=\hat{h}_{n, m}\left(\mu_{m, t_{i}}, v_{n, t_{i}}\right)$ is the estimated $N \times M$ MIMO channel.

During the training sessions, the receiver sorts the modes according to the resulting channel qualities and stores them. Within a certain number of training sessions, the receiver gathers a list of antenna modes with relatively good channel qualities. At the end of the training sessions, the receiver reports the list to the transmitter implying that the upcoming transmissions will employ only the modes within the list. Thus, a lower number of antenna modes will be trained, and hence the training overhead will be reduced. Such a procedure also alleviates the estimation problem when some or all of selected trained modes in $S_{b_{n}}$ experience a deep fade as the transmitter updates the modes to be trained for the next training round. Note that the list may be updated whenever the channel qualities for the selected list of antenna modes become worse. To do so, the transmitter and/or receiver may request to reset the list and restart the training procedure with the initially agreed upon list.

\section{Joint Estimation and Prediction for MR-MIMO Channels}

The overhead due to the training mechanisms described in Section III-C may be prohibitive in the presence of many antenna modes. In order to reduce this overhead, we utilize the channel representations developed in the previous section. Using the analysis developed in Section III-A, one can exploit the presence of correlations among the channels at different antenna modes. For instance, a subset of antenna modes can be selected to train the channel to obtain an estimate of $\overline{\boldsymbol{H}}_{n, m}$ and then the channel gains for all other modes can be predicted. To that end, let us assume that $F$ antenna modes are selected for channel training. At a given instance, the CSI for these $F$ modes can be written from (9) as

$$
\boldsymbol{h}_{n, m}=\boldsymbol{\Gamma} \operatorname{vec}\left(\overline{\boldsymbol{H}}_{n, m}\right),
$$

with $\boldsymbol{\Gamma}=\left[\boldsymbol{\gamma}_{\nu}^{T} \otimes \boldsymbol{\alpha}_{\mu_{1}}^{H} \quad \cdots \quad \boldsymbol{\gamma}_{\nu}^{T} \otimes \boldsymbol{\alpha}_{\mu_{F}}^{H}\right]^{T}$, and $\boldsymbol{h}_{n, m}=$ $\left[h_{n, m}\left(\mu_{1}, v\right), \ldots, h_{n, m}\left(\mu_{F}, v\right)\right]^{T}$. By using (9), the same procedure can be repeated for all the remaining $L_{\mu}-F$ antenna modes to obtain the corresponding channel state estimates

$$
\boldsymbol{h}_{n, m}^{c}=\left[h_{n, m}\left(\mu_{F+1}, v\right), \ldots, h_{n, m}\left(\mu_{L_{\mu}}, v\right)\right]^{T} .
$$


Different prediction algorithms can be used. For instance, by using the least squares (LS) criterion, we can represent $\boldsymbol{h}_{n, m}^{c}$ in terms of the channel realizations from the training set $\boldsymbol{h}_{n, m}$ as

$$
\boldsymbol{h}_{n, m}^{c}=\boldsymbol{\Gamma}^{c}\left(\boldsymbol{\Gamma}^{H} \boldsymbol{\Gamma}\right)^{-1} \boldsymbol{\Gamma}^{H} \boldsymbol{h}_{n, m}
$$

with $\boldsymbol{\Gamma}^{c}=\left[\boldsymbol{\gamma}_{\nu}^{T} \otimes \boldsymbol{\alpha}_{\mu_{F+1}}^{H} \ldots \boldsymbol{\gamma}_{\nu}^{T} \otimes \boldsymbol{\alpha}_{\mu_{L_{\mu}}}^{H}\right]^{T}$.

When $F_{t} F_{r}>F$, which is typical for the underlying MR-MIMO systems, the system is under-determined since we have more unknown variables than the available observations. Therefore, the LS solution may not perform well and the performance may get worse as the number of untrained modes $L_{\mu}-F$ increases well beyond the number of trained modes. Furthermore, the errors inherited from the estimation of trained modes may become more critical for the prediction performance. On the other hand, as described in Section III-A, channel correlations exist among different antenna modes, and thus, the problem can be formulated as an MMSE prediction problem as described next.

1) MMSE Estimation and Prediction: We can utilize the inherent correlation between the channel realizations of different antenna modes for the same channel propagation state. Using MMSE to obtain $\hat{\boldsymbol{h}}_{n, m}^{c}$ from the received signal $\boldsymbol{r}(\boldsymbol{\mu}, \boldsymbol{v})$ in (14), we obtain the channel estimates as

$$
\begin{aligned}
\hat{\boldsymbol{h}}_{n, m}^{c} & =\boldsymbol{R}_{\boldsymbol{h}^{c} \boldsymbol{h}}\left(\boldsymbol{R}_{\boldsymbol{h} \boldsymbol{h}}+\boldsymbol{P}^{-1} \boldsymbol{R}_{z z} \boldsymbol{P}^{-H}\right)^{-1} \boldsymbol{P}^{-1} \boldsymbol{r} \\
& =\boldsymbol{R}_{\boldsymbol{h}^{c} \boldsymbol{h}} \boldsymbol{R}_{\boldsymbol{h} \boldsymbol{h}}^{-1} \hat{\boldsymbol{h}},
\end{aligned}
$$

which clearly indicates that the estimates for $\boldsymbol{h}_{n, m}^{c}$ can be predicted from the estimates of $\boldsymbol{h}_{n, m}$ using the cross-correlations among the channel realizations of the trained modes and the remaining ones. The error covariance matrix for this estimator is given by

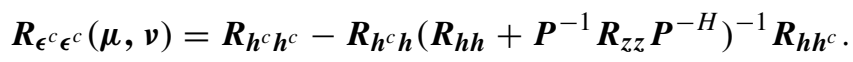

Using the estimation and prediction covariance matrices in (15) and (19), the overall mean square error for channel estimation and prediction from the received signal can be expressed as

$$
\begin{aligned}
\boldsymbol{\epsilon} & =\frac{1}{L_{\mu}} \operatorname{Tr}\left(\boldsymbol{R}_{\boldsymbol{h} \boldsymbol{h}}+\boldsymbol{R}_{\boldsymbol{h}^{c} \boldsymbol{h}^{c}}\right) \\
& =1-\frac{1}{L_{\mu}} \operatorname{Tr}\left(\left(\boldsymbol{R}_{\boldsymbol{h} \boldsymbol{h}}^{2}+\boldsymbol{R}_{\boldsymbol{h} \boldsymbol{h}^{c}} \boldsymbol{R}_{\boldsymbol{h} \boldsymbol{h}^{c}}^{H}\right)\left(\boldsymbol{R}_{\boldsymbol{h} \boldsymbol{h}}+\boldsymbol{P}^{-1} \boldsymbol{R}_{z z} \boldsymbol{P}^{-H}\right)^{-1}\right) .
\end{aligned}
$$

We observe that the selection of $F$ training modes from $L_{\mu}$ candidates can be cast as an optimization problem to minimize $\epsilon_{t}$, which can be simplified to

$$
\begin{aligned}
\left(\boldsymbol{\mu}^{*}, \boldsymbol{v}^{*}\right)= & \arg \max _{\boldsymbol{\mu}, \boldsymbol{v}} \operatorname{Tr}\left(\left(\boldsymbol{R}_{\boldsymbol{h} \boldsymbol{h}}^{2}+\boldsymbol{R}_{\boldsymbol{h} \boldsymbol{h}^{\boldsymbol{c}}} \boldsymbol{R}_{\boldsymbol{h} \boldsymbol{h}^{c}}^{H}\right)\right. \\
& \left.\times\left(\boldsymbol{R}_{\boldsymbol{h} \boldsymbol{h}}+\boldsymbol{P}^{-1} \boldsymbol{R}_{z z} \boldsymbol{P}^{-H}\right)^{-1}\right) .
\end{aligned}
$$

At high SNRs, e.g., as $\boldsymbol{R}_{z z} \rightarrow 0$, we can further simplify (20) as

$$
\left(\boldsymbol{\mu}^{*}, \boldsymbol{v}^{*}\right)=\arg \max _{\boldsymbol{\mu}, \boldsymbol{v}} \operatorname{Tr}\left(\boldsymbol{R}_{\boldsymbol{h} \boldsymbol{h}^{c}}^{H} \boldsymbol{R}_{\boldsymbol{h} \boldsymbol{h}}^{-1} \boldsymbol{R}_{\boldsymbol{h} \boldsymbol{h}^{c}}\right) .
$$

The optimization in (21) is a combinatorial problem where the selection of $F$ modes amounts to finding an

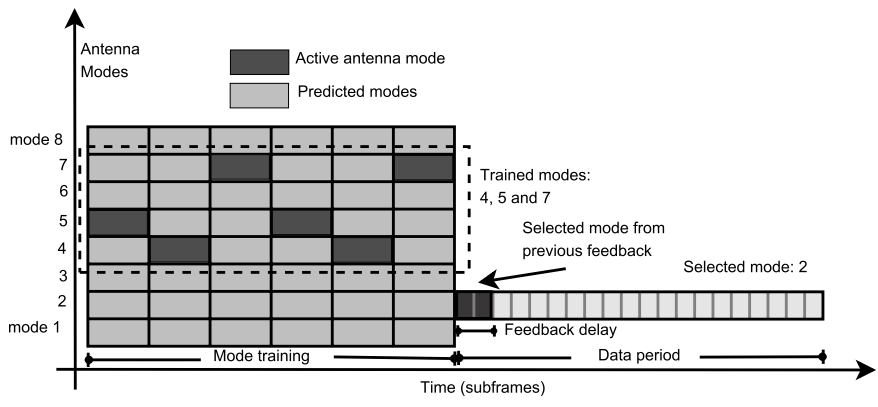

Fig. 5. Example MRA training over time.

$F \times F$ sub-matrix, $\boldsymbol{R}_{\boldsymbol{h} \boldsymbol{h}}$, the resulting $F \times L_{\mu}-F$ crosscorrelation matrix, $\boldsymbol{R}_{\boldsymbol{h} \boldsymbol{h}^{c}}$ from the $L_{\mu} \times L_{\mu}$ correlation matrices evaluated for the $L_{\mu}$ candidate modes. For a small number of candidate modes, this search can be performed quickly. In case of a large number of modes, random search methods can be utilized to determine a sub-optimal but efficient solution (see, e.g. [41]).

Once the channel for each mode are estimated/predicted, the receiver reports the selected antenna mode(s) to the transmitter after a feedback delay. Let $T_{f b}$ denote the number of channel uses required for transmitter to receive this report after the last training symbol is transmitted. During this time, the transmitter and the receiver may agree to employ a specific mode, for instance, the most recent mode reported since it is likely to be still a good choice for slow fading channels. This feedback delay is typically very small and for $T_{f b} \ll T_{c}$, its impact on the performance will be negligible. Fig. 5 depicts a typical estimation/prediction timing using an OFDM frame structure where at any transmission subframe, one of the modes is used for probing the channel training. In the example, 8 different modes exist, and 3 of them are selected for training. Note that before the actual training starts with the selected modes, the system may employ the procedure described in Section III-C to train all the 8 modes and obtain estimates for the channel covariance matrices to be employed during the MMSE prediction step.

Number of Training Modes: We next develop a technique to determine a suitable value for the number of modes $(F)$ to be trained. Since the estimates of the wireless channel gains are valid within the coherence time of the channel, let us consider a training within a coherence block of $T_{c}$ symbols during which the channel remains fixed. Assuming that each mode is trained for $\beta \in\left\{1,2, \ldots,\left\lceil\frac{T_{c}}{F}\right\rceil\right\}$ symbols, we have $L_{p}=\beta F$ symbols where the transmission is performed via the trained modes, and $T_{c}-\beta F$ symbols with transmission employing the best mode, $\mu^{*}$. The average achievable rate for this transmission can be approximated by (using Jensen's inequality along with the Shannon capacity for a fading channel) [20]

$$
\begin{aligned}
C(F) \lesssim & \frac{\beta}{T_{c}} \sum_{i=1}^{F} \log _{2}\left(1+\frac{1}{\sigma_{z}^{2}} E\left\{\left|h\left(\mu_{i}\right)\right|^{2}\right\}\right)+\left(1-\frac{\beta F}{T_{c}}\right) \\
& \times \log _{2}\left(1+\frac{1}{\sigma_{z}^{2}} E\left\{\left|h\left(\mu^{*}\right)\right|^{2}\right\}\right) \\
\text { s.t. } \mu^{*}= & \arg \max _{\mu \in\left\{\mu_{1}, \ldots, \mu_{L_{\mu}}\right\}}|\hat{h}(\mu)|^{2} .
\end{aligned}
$$


where $\mu^{*}$ is obtained at the end of mode training session. With the MMSE based estimation/prediction method, we have $\hat{h}(\mu)=h(\mu)+e(\mu)$ where the estimation error $e\left(\mu_{i}\right) \sim$ $C N\left(0, \sigma_{e_{i}}^{2}\right)$ with $\sigma_{e_{i}}^{2}=\left[\boldsymbol{R}_{\boldsymbol{\epsilon} \epsilon}\right]_{i, i}$, for $i \in\{1, \ldots, F\}$ and

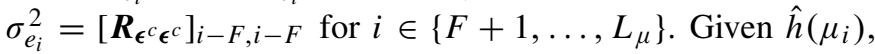
$i=1, \ldots, L_{\mu}$, we can express $h\left(\mu^{*}\right)$ as

$$
h\left(\mu^{*}\right)=\sum_{i=1}^{L_{\mu}} h\left(\mu_{i}\right) \prod_{n \neq i} I\left(\left|\hat{h}\left(\mu_{i}\right)\right|^{2} \geq\left|\hat{h}\left(\mu_{n}\right)\right|^{2}\right)
$$

where $I(\cdot)$ is the indicator function, i.e., $I(A)=1$ if the condition defined by $A$ is satisfied. Using the total expectation theorem, we can write

$$
\begin{aligned}
E\left\{\left|h\left(\mu^{*}\right)\right|^{2}\right\} & \\
= & E\left\{\sum _ { i = 1 } ^ { L _ { \mu } } E \left\{\left|h\left(\mu_{i}\right)\right|^{2}\right.\right. \\
& \left.\left.\times \prod_{n \neq i} I\left(\left|\hat{h}\left(\mu_{i}\right)\right|^{2} \geq\left|\hat{h}\left(\mu_{n}\right)\right|^{2}\right) \mid \hat{h}\left(\mu_{i}\right)\right\}\right\} \\
= & E\left\{\sum_{i=1}^{L_{\mu}} E\left\{\left|h\left(\mu_{i}\right)\right|^{2} \mid \hat{h}\left(\mu_{i}\right)\right\}\right. \\
& \left.\times E\left\{\prod_{n \neq i} I\left(\left|\hat{h}\left(\mu_{i}\right)\right|^{2} \geq\left|\hat{h}\left(\mu_{n}\right)\right|^{2}\right) \mid \hat{h}\left(\mu_{i}\right)\right\}\right\}
\end{aligned}
$$

where (25) follows as $h\left(\mu_{i}\right)$ and $\left.I\left(\left|\hat{h}\left(\mu_{i}\right)\right|^{2} \geq \mid \hat{h}\left(\mu_{j}\right)\right)\right|^{2}$ are conditionally independent given $\hat{h}\left(\mu_{i}\right)$. This expression can be evaluated numerically, however, to obtain a tractable analytical solution, let us consider the case where $h\left(\mu_{i}\right) \sim \mathcal{C N}\left(0, \sigma_{h_{i}}^{2}\right)$, with $\sigma_{h_{i}}^{2}=\left[\boldsymbol{R}_{\boldsymbol{h} \boldsymbol{h}}\right]_{i, i}, i=1, \ldots, F$, and $\sigma_{h_{i}}^{2}=\left[\boldsymbol{R}_{\boldsymbol{h}^{c}} \boldsymbol{h}^{c}\right]_{i-F, i-F}$, $i=F+1, \ldots, L_{m}$, are mutually independent. Then, we can write

$$
\begin{aligned}
E\left\{\left|h\left(\mu^{*}\right)\right|^{2}\right\} \approx & E\left\{\sum_{i=1}^{L_{\mu}} E\left\{\left|h\left(\mu_{i}\right)\right|^{2} \mid \hat{h}\left(\mu_{i}\right)\right\}\right. \\
& \left.\times \prod_{n \neq i} E\left\{I\left(\left|\hat{h}\left(\mu_{i}\right)\right|^{2} \geq\left|\hat{h}\left(\mu_{n}\right)\right|^{2}\right) \mid \hat{h}\left(\mu_{i}\right)\right\}\right\} \\
= & \sum_{i=1}^{L_{\mu}} \int Q_{i}(y) \prod_{n \neq i}\left(1-e^{-\frac{|y|^{2}}{\sigma_{h_{n}}^{2}+\sigma_{e_{n}}^{2}}}\right) \\
& \times \frac{1}{\pi\left(\sigma_{h_{i}}^{2}+\sigma_{e_{i}}^{2}\right)} e^{-\frac{|y|^{2}}{\sigma_{h_{i}}^{2}+\sigma_{e_{i}}^{2}}} d y
\end{aligned}
$$

with

$$
\begin{aligned}
Q_{i}(y)= & \int|h|^{2} \frac{\sigma_{h_{i}}^{2}+\sigma_{e_{i}}^{2}}{\pi \sigma_{h_{i}}^{2} \sigma_{e_{i}}^{2}} \\
& \times \exp \left(\frac{|y|^{2}}{\sigma_{h_{i}}^{2}+\sigma_{e_{i}}^{2}}-\frac{|y-h|^{2}}{\sigma_{e_{i}}^{2}}-\frac{|h|^{2}}{\sigma_{h_{i}}^{2}}\right) d h .
\end{aligned}
$$

Substituting (26) and $E\left\{\left|h\left(\mu_{i}\right)\right|^{2}\right\}=\sigma_{h_{i}}^{2}$ in (22), we can finally calculate the expected rate using $F$ trained modes.
We note that (22) quantifies the trade-off between the estimation/prediction performance and the resulting achievable rate. For small $F$, one will use less resources for mode training, however, due to the inferior prediction performance, the selected mode $\mu^{*}$ is more likely to be suboptimal, and transmission with this mode will contribute less to the average rate. On the other hand, for large $F$, more resources will be employed for the training session which reduces prediction errors, and thus improves the mode selection performance. However, in this case, less resources will be employed for transmission with the improved mode.

A numerical example for optimization of $F$ based on (22) is provided in Fig. 6. Here, (22) is evaluated via Monte-Carlo integration techniques to calculate the average throughput gain defined as $100\left(\frac{C(F)}{C(1)}-1\right)$, i.e., the percentage gain with respect to the case of $F=1$ trained modes. We study two cases with $L_{\mu}=9$ and $L_{\mu}=20$ available modes and for each case we consider various $T_{c}$ values corresponding to different levels of mobility. It is seen that higher coherence time values of $T_{c}$ allows for larger $F$ indicating that one can improve the expected throughput by training more modes. On the other hand, for lower $T_{c}$ values, for example, when $T_{c}=18,36$ for $L_{\mu}=9$ and $T_{c}=40,80$ for $L_{\mu}=20$ modes, it is seen that the expected gains increase up to a certain point and then the gains start to decrease with increasing $F$.

The mode selection criteria described above relies on the average covariance matrices and thus the average MSE performance. Thus, for selection of mode set and $F$ in this manner, we attempt to optimize the average estimation performance. We note that this scheme can be further improved by monitoring the channel gains for trained and nontrained modes, and then using the intelligent mode update scheme described in Section III-C where the modes with higher channel gains (obtained from earlier estimation/prediction sessions) may be selected for subsequent training. In this case, the trained modes will provide improved estimation performance, and in return, the channel prediction will be more reliable.

2) Estimation of Covariance Matrices: For realistic channel estimation schemes used in practice, the covariance matrices should also be estimated from the observations [42]. Here, we assume a sample covariance matrix estimation method where the instantaneous observations and channel estimates are obtained at each OFDM subframe transmission and averaged via an exponential filter over time. Let us express the channel between the transmit antenna- $m$ and the receive antenna- $n$ at symbol time $l=1,2, \ldots$, for the modes $\mu_{1}, \ldots, \mu_{F}$, as an $F \times 1$ vector $\boldsymbol{h}_{n, m, l}(\boldsymbol{\mu}, v)=$ $\left[h_{n, m, l}\left(\mu_{1}, \nu\right), \ldots, h_{n, m, l}\left(\mu_{F}, \nu\right)\right]^{T}$. The sample covariance matrix can be estimated via a first-order IIR filter [42]

$$
\overline{\hat{\boldsymbol{R}}}_{\boldsymbol{h \boldsymbol { h }}}(l+1)=\zeta \overline{\boldsymbol{\boldsymbol { R }}}_{\boldsymbol{h \boldsymbol { h }}}(l)+(1-\zeta) \hat{\boldsymbol{h}}_{n, m, l}(\boldsymbol{\mu}, v) \hat{\boldsymbol{h}}_{n, m, l}^{H}(\boldsymbol{\mu}, v)
$$

where $\zeta$ is a constant determining the memory of the averaging filter over time.

Temporal Smoothing via a Kalman Filter: The MMSE estimation/prediction described above utilizes spatial domain correlations to determine and/or smooth the channel estimates. 


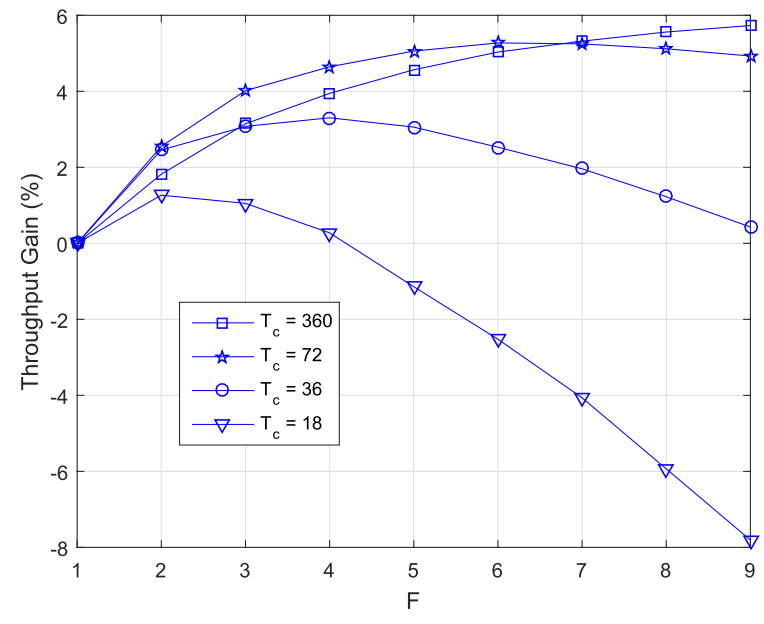

(a)

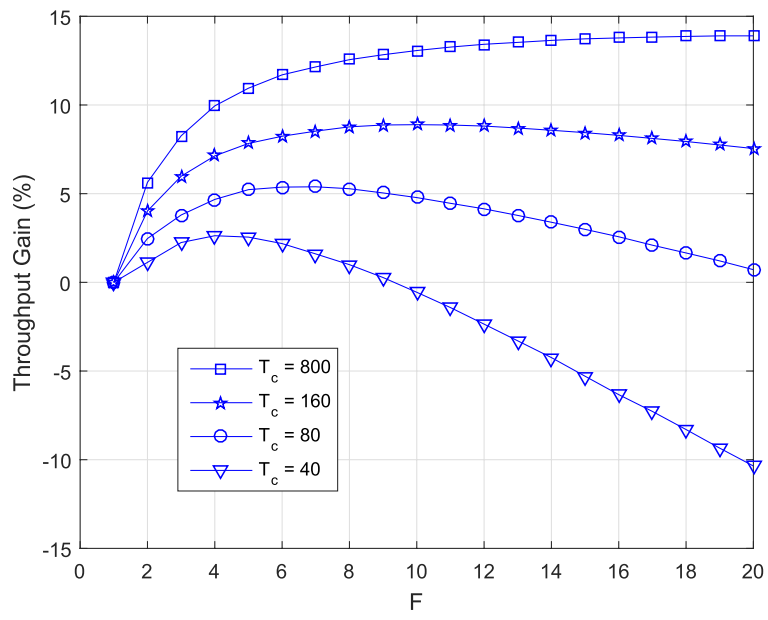

(b)

Fig. 6. Average throughput gain, $100\left(\frac{C(F)}{C(1)}-1\right)$ for $\beta=2$, and (a) $L_{\mu}=9$ modes, (b) $L_{\mu}=20$ modes.

From Fig. 5, it is clearly seen that the time between two successive training periods for a given antenna mode may get larger if the number of modes used for training increases. In this case, the temporal correlations for the channel samples during this period may be optimally estimated via an augmented Kalman filter where the temporal correlations and the channel values for those subframes during which no training signal is transmitted are estimated. To that end, we consider a first-order state-space representation for the temporal variations of the channel, namely,

$$
\boldsymbol{h}_{n, m, l+1}(\boldsymbol{\mu}, v)=\mathcal{T}(l+1, l) \boldsymbol{h}_{n, m, l}(\boldsymbol{\mu}, v)+v_{1}(l)
$$

where $\mathcal{T}(l+1, l)$ denotes the channel state transmission matrix from one symbol time to the next, and $v_{1}(l)$ denotes the state estimation error arising from modeling. The channel estimates are smoothed temporally over time using the received signals

$$
r_{n, l}(\mu, \nu)=h_{n, m, l}(\mu, \nu) p_{m, l}+z_{n, l},
$$

$l=l_{0}, l_{0}+\Delta, l_{0}+2 \Delta, \ldots$, where $\Delta$ denotes the time interval between two successive training for a given mode $\mu \in\left\{\mu_{1}, \ldots, \mu_{F}\right\}$.

Note that the Kalman filter update is an iterative approach to reach MMSE solution. Hence, its application in the proposed mode estimation/prediction scheme can be imagined as obtaining an improved MMSE solution to the channel estimation/prediction scheme by utilizing the temporal correlations. With the Kalman filter updates, the estimates of the channel state information for all the modes are obtained and the best mode is selected at the end of each training session. The selected mode is employed during the following data transmission period.

\section{TRANSMISSION WITH MRAS}

\section{A. Signaling With OFDM Modulation}

Due its widespread use in state-of-the art wireless technologies [43]-[45], we consider an OFDM based MIMO transmission in our simulations [46]. Assuming a cyclic prefix $\left(T_{C P}\right)$ of sufficient duration, i.e., $T_{C P} \geq \max _{j, i} \tau_{j, i}$, and perfect time and frequency synchronization, we can express the input-output signal relation from antenna- $m$ to antenna- $n$, at time $t=i T_{s}, i=1,2, \ldots$, and at subcarrier $f_{c}+k \Delta f$, $k=-N_{f} / 2, \ldots, N_{f} / 2-1$, as

$$
r_{n, i, k}\left(\boldsymbol{\mu}, v_{n}\right)=\sum_{m=1}^{M} h_{n, m, i, k}\left(\mu_{m}, v_{n}\right) s_{m, i, k}+z_{n, i, k}
$$

where $T_{s}=\frac{1}{\Delta f}+T_{C P}$ is the total OFDM symbol duration including the cyclic prefix, $f_{c}$ is the carrier frequency, $\Delta f$ is the subcarrier spacing, and $N_{f}$ is the DFT size of the OFDM signal. Here, $z$ denotes the additive circularly symmetric zeromean complex Gaussian noise with variance $\sigma^{2} / 2$ per dimension, and $s_{m, i, k}$ is the signal transmitted from the $m^{\text {th }}$ antenna at symbol $i$ and subcarrier $k$. The transmit symbol energies are normalized to unity so that the transmit SNR is defined as $\operatorname{TxSNR}=\frac{1}{\sigma^{2}}$.

The MRA in all of the examples below has a $3 \times 3$ parasitic pixel surface with 12 interconnecting switches (see Fig. 1.b in Section II-A). The principles on the design and optimization of a similar MRA is available in [24]. A uniform linear array of $M=N=2$ MRAs with $\lambda / 2$ interelement spacing is assumed for the MR-MIMO system created by these MRAs. ${ }^{1}$ For each MRA, several modes of operation are generated using the Genetic Algorithm as described in [5]. The full-wave analysis tool HFSS [47] is employed to generate 3D complex radiation patterns.

\section{B. Performance With MRAs}

In this section, we analyze the channel estimation algorithms proposed so far via simulations. We consider the frame numerology from 3GPP LTE-A where the minimum resource block (subframe) for scheduling contains 12 subcarriers and 14 OFDM symbols [48]. We set the subcarrier spacing as $\Delta_{f}=15 \mathrm{kHz}$. With $T_{C P}=4.7 \mu s$ cyclic prefix length, we have a symbol time of $71 \mu \mathrm{s}$. Thus each subframe is $1 \mathrm{~ms}$.

\footnotetext{
${ }^{1}$ We note that the analysis that follows is not limited to this specific MIMO antenna geometry, and the arguments can be generalized with ease.
} 


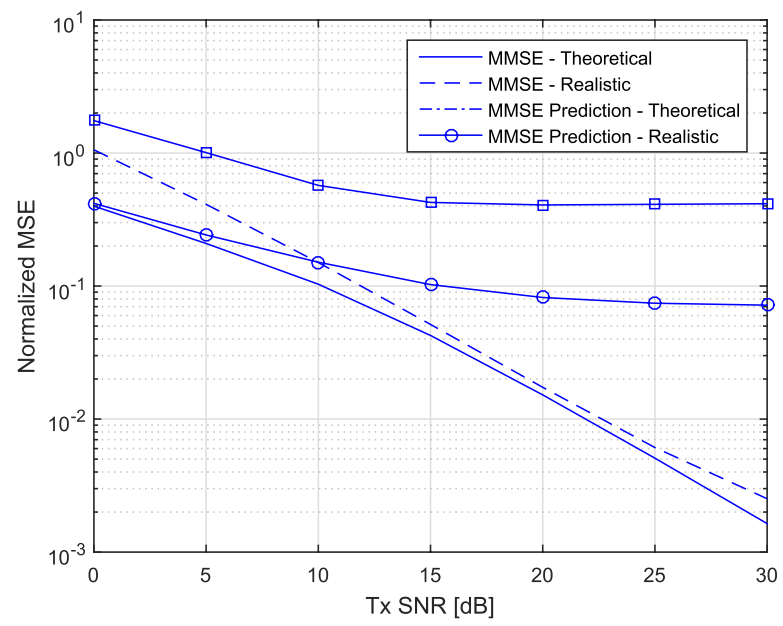

(a)

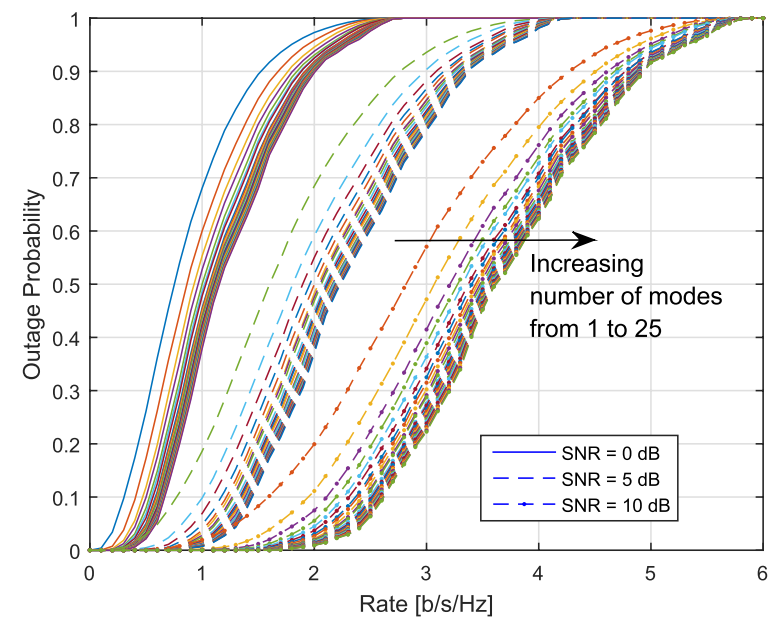

(c)

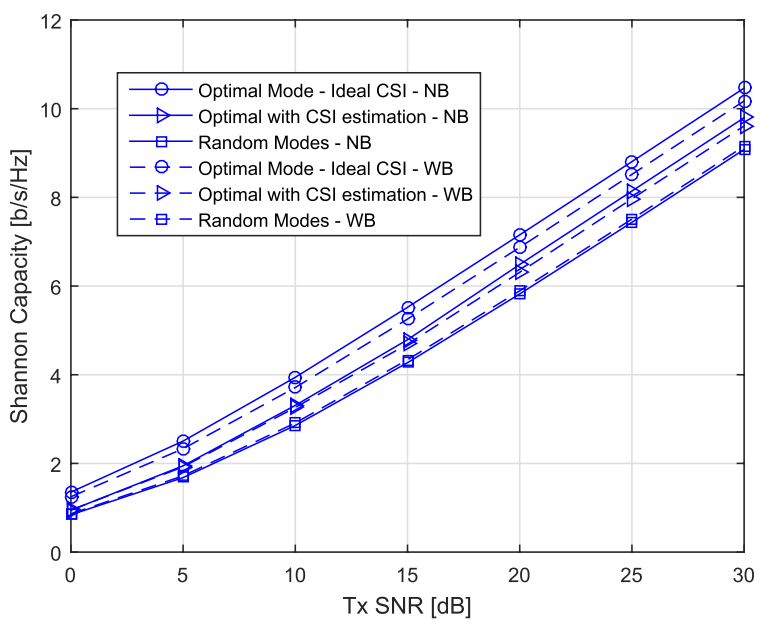

(b)

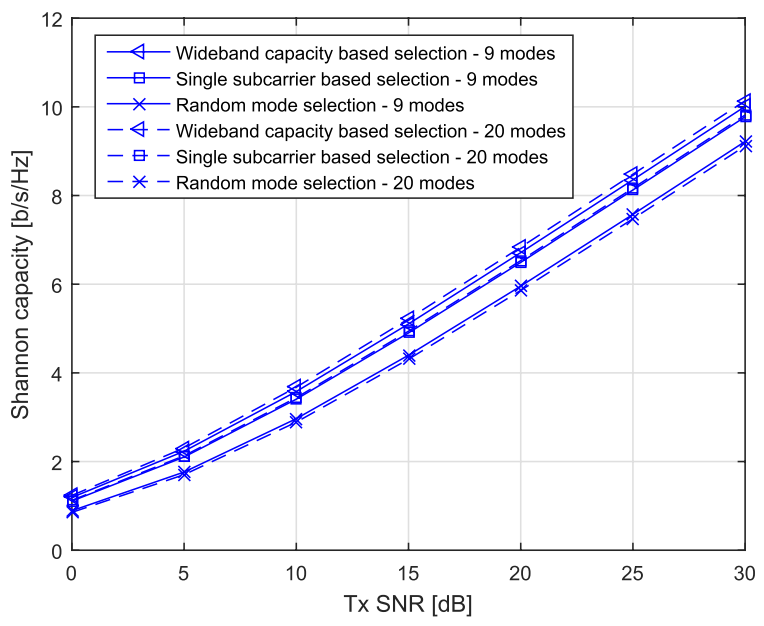

(d)

Fig. 7. User speed $=1 \mathrm{~km} / \mathrm{h}$. (a) Theoretical and realistic channel estimation error comparison for narrowband transmission, (b) Shannon capacities with ideal and realistic CSI estimation, (c) outage probabilities as number of antenna modes is varied, (d) channel capacities with narrowband and wideband based mode selection.

We assume that a periodic pilot signal is transmitted every 7 symbols over time and every 6 subcarrier over frequency which amounts to 4 pilot symbols per subframe. For the double directional channel model, we assume the spatial channel model from Winner+ B1 channel [35] with 16 taps, each with 20 non-resolvable subpaths. Since we assume spatially orthogonal pilots, we focus on a single channel from the transmit antenna- $m$ to the receive antenna- $n$.

Figs. 7 and 8 illustrate performance of an MRA at a user speed of $1 \mathrm{~km} / \mathrm{h}$. To obtain these results, $L_{\mu}=9$ candidate modes out of the 25 are selected, which reflects a snapshot of the intelligent mode update based solution described in Section III. During the initial training period of 450 subframes, all 9 modes are trained periodically such that in each subframe, one of the modes is used during transmission. Thus, each mode is trained with 50 subframes separated by 9 subframes. During this period, the sample covariance matrices of channels among different modes are obtained using the approaches in III-C.1 and III-D. After the initial training period, $F=3$ modes are selected for the following training periods, while the remaining $L_{\mu}-F=6$ modes are predicted using the MMSE approach along with the temporal smoothing based on the Kalman filtering procedure described in the previous section. The selection of modes to be trained within the 9 modes is performed using (21). The periodic training session parameters, e.g., the number of OFDM symbols for training session $\left(L_{p}\right)$ and data transmission $\left(T_{c}-L_{p}\right)$, are varied to find the values with the best system performance, which turns out to be $L_{p}=18$ ( 9 subframes) and $T_{c}-L_{p}=54$ (27 subframes), respectively, for this example.

The mean square error values in Fig. 7.a for estimation and prediction are averaged over the 3 training modes and 6 prediction modes, respectively, to indicate an average performance. Clearly, the performance for both ideal and realistic CSI estimation is superior to the performance for prediction modes as expected since the channel prediction is based only 


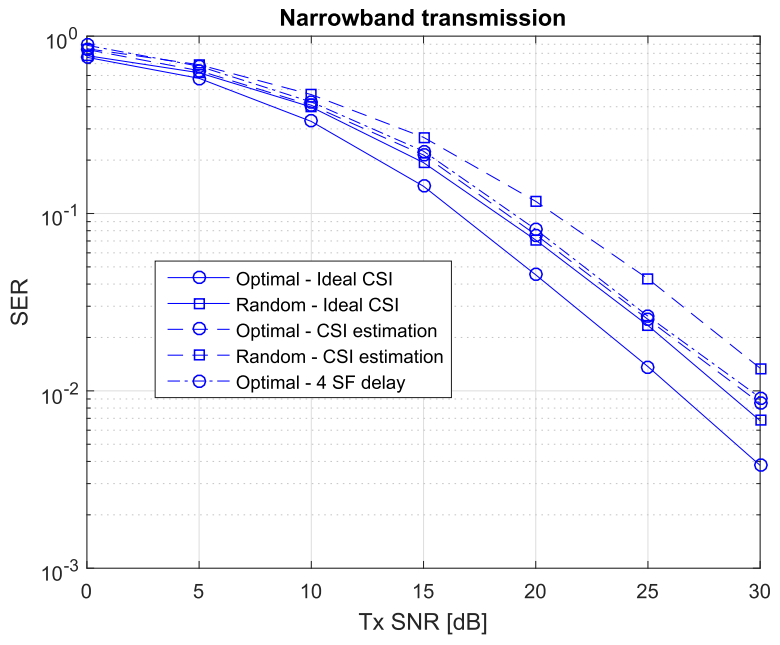

(a)

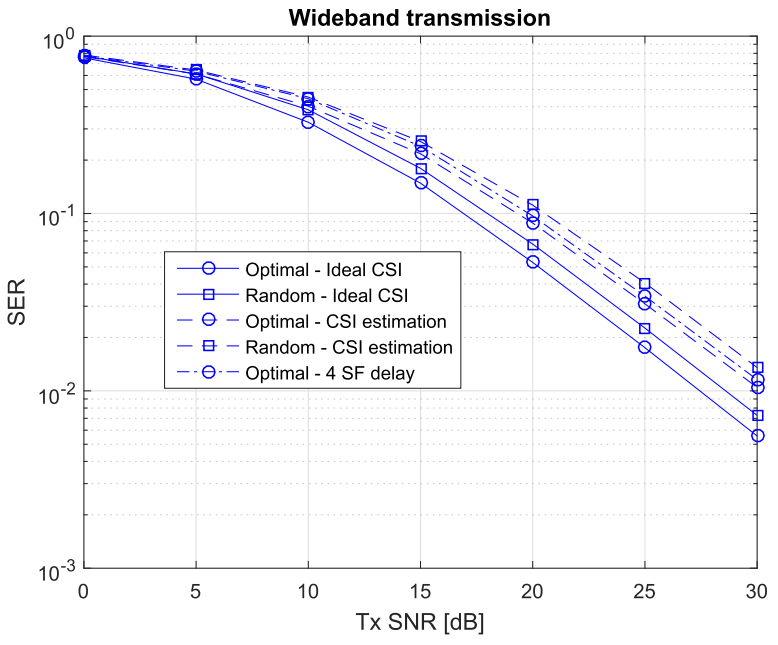

(b)

Fig. 8. Symbol error rate (a) narrowband (b) wideband transmission. For both cases, mode selection based on a single subcarrier is performed using (21).

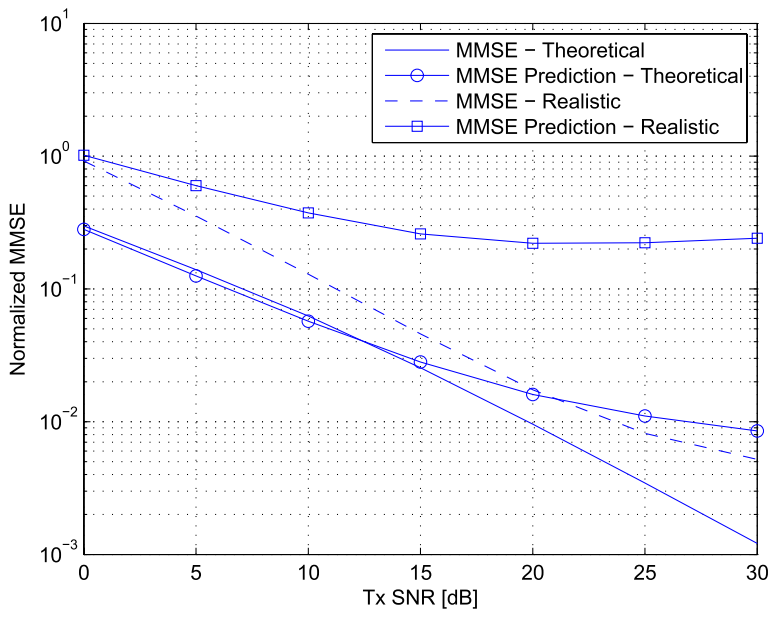

(a)

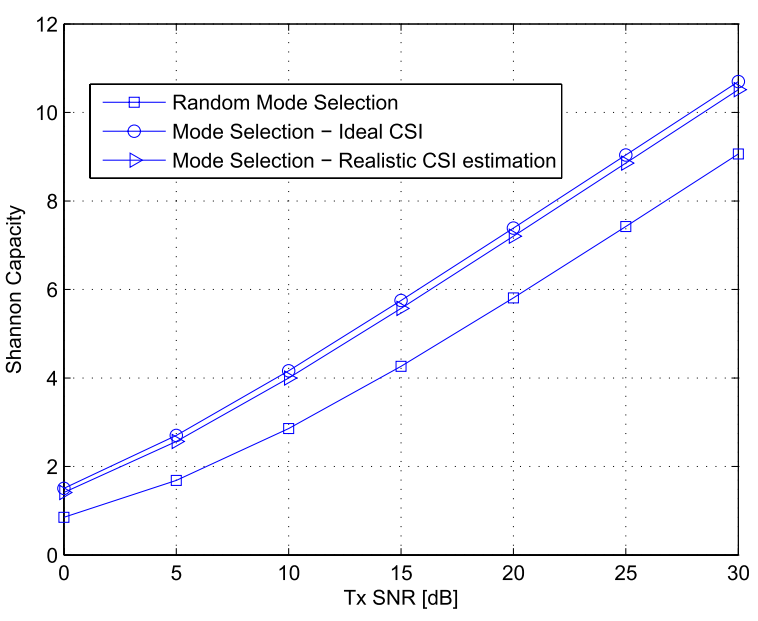

(b)

Fig. 9. Out of 25 antenna modes, 20 modes are selected randomly for transmission. Fixed receive mode. For realistic CSI estimation/prediction, after an initial training period of all 20 modes, 4 of them are randomly selected for further training while other 16 modes are predicted using MMSE approach. $1 \mathrm{~km} / \mathrm{h}$ user speed. (a) Mean square error results for CSI estimation and prediction, (b) Shannon capacity results for CSI estimation and prediction.

on the trained modes and the available correlations resulting from the modes of operation. However, as shown from the achievable rate performance results illustrated in Fig. 7.b, the theoretical SNR gains from the ideal mode selection are around $3 \mathrm{~dB}$ and the practical mode selection based on the proposed estimation/prediction process is around $2 \mathrm{~dB}$ compared to a random mode selection scheme. Using the symbol error rates for uncoded transmission with 16-QAM (see Figs. 8.a and 8.b), it is also seen that the MRA with mode selection achieves up to $3 \mathrm{~dB}$ SNR gains. Note that these gains are obtained with identical overhead for both MRA and fixed antenna systems. The curves marked as random mode selection in Figs. 7.b and 7.d indicate the performance of a link with an (arbitrarily picked) fixed antenna mode.

In order to evaluate the impact of the number of modes on the system performance, we depict the outage probabilities for various scenarios in Fig. 7.c. Here, for each SNR and for each curve at a given SNR, the outage probabilities are calculated for $F$ modes, $F=1, \ldots, 25$, and for each $F$, the outage probability is averaged over 1000 realizations of $\mathcal{S}=\left\{\mu_{1}, \ldots, \mu_{F}\right\}$. Hence, the results indicate an average performance obtained by employing an MRA with $F$ active modes of operation. It is clearly seen that significant gains can be achieved using MRAs. For example, $10 \%$ outage rate improves by $125 \%$ and $85 \%$ at $\mathrm{SNR}=0 \mathrm{~dB}$ and $5 \mathrm{~dB}$, respectively, with 25 antenna modes. It is also seen, as one would expect, that only diminishing returns are available as the number of modes is increased. This implies that one may get most of the gains out of an MRA system using a proper mode subset selection.

In Fig. 7.d, a comparison between performance for two different mode selection mechanisms is provided. In the single subcarrier based mode selection scheme, the gain of the channel at a single subcarrier is employed, while in the wideband capacity based selection, the antenna mode with the highest wideband capacity averaged over all pilot tones is used at any 


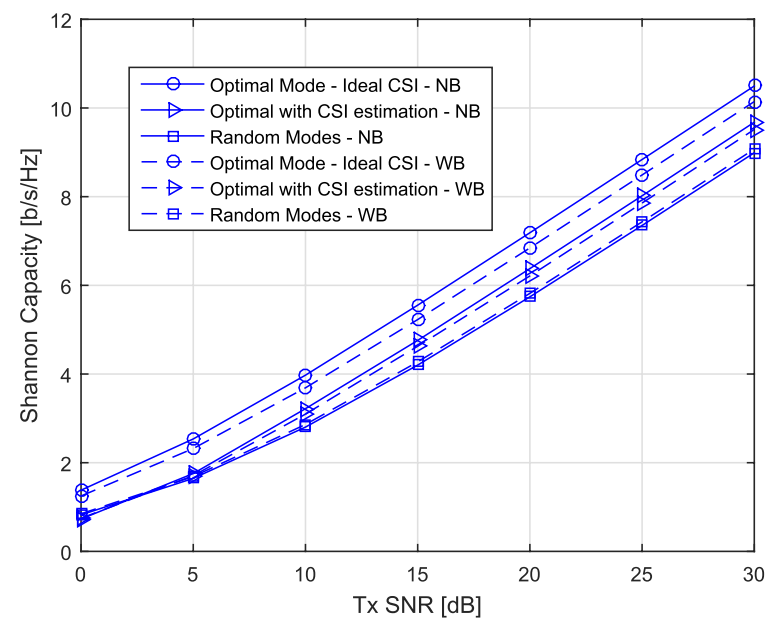

(a)

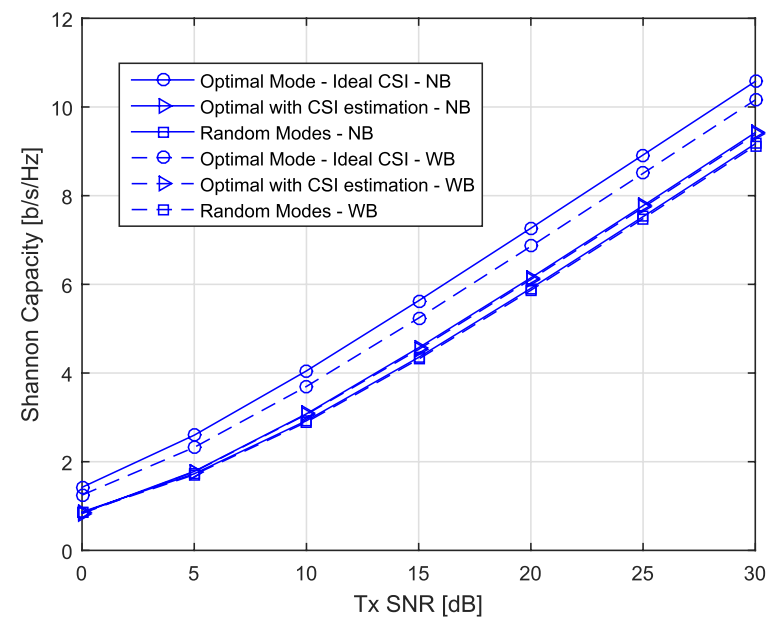

(b)

Fig. 10. Shannon rate comparisons for (a) $5 \mathrm{~km} / \mathrm{h}$ (b) $20 \mathrm{~km} / \mathrm{h}$. For both cases, mode selection based on a single subcarrier is performed using (21).

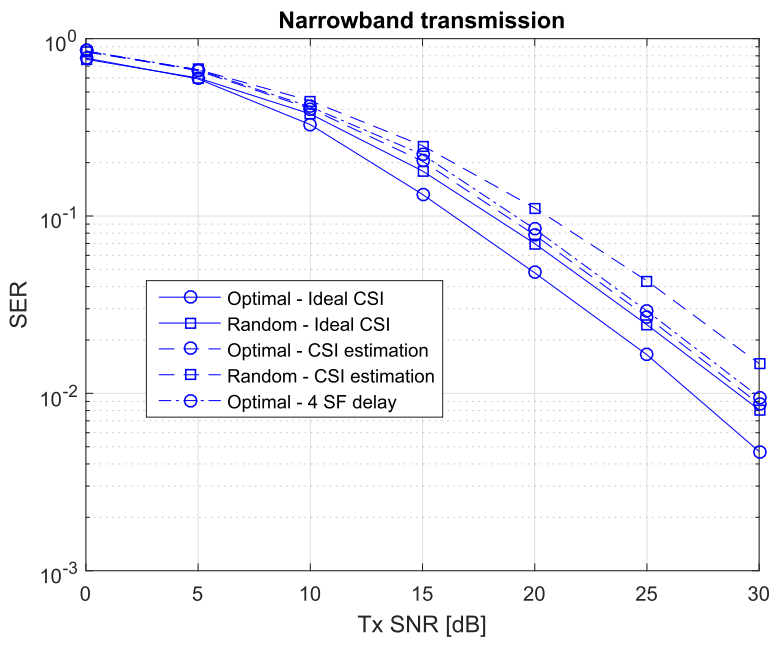

(a)

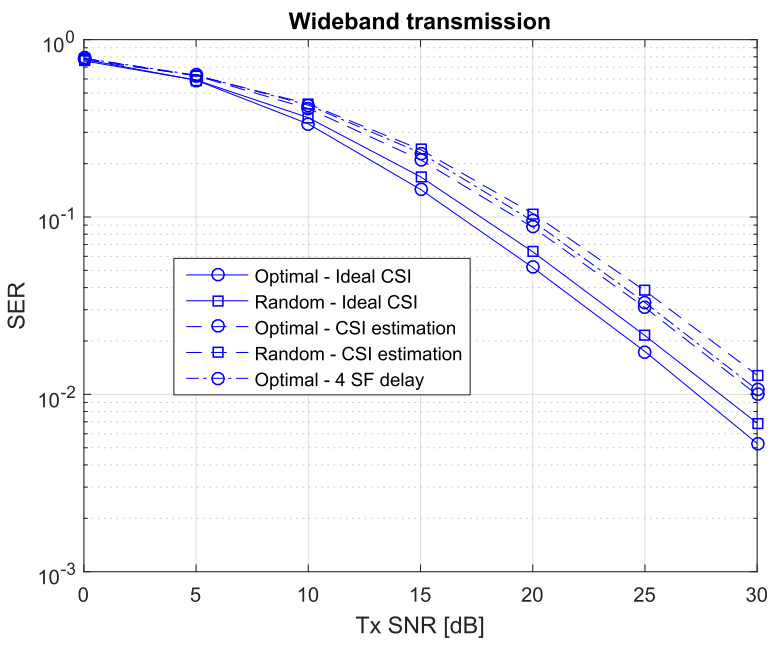

(b)

Fig. 11. Symbol error rate for $5 \mathrm{~km} / \mathrm{h}$ (a) narrowband (b) wideband transmission. For both cases, mode selection based on a single subcarrier is performed using (21).

time. The results are obtained for two different MRAs with 9 and 20 modes. From Fig. 7.d, we observe only a slight degradation (of less than $1 \mathrm{~dB}$ ) with the suboptimal mode selection based on a single subcarrier for both cases.

In Fig. 8, we also illustrate the impact of feedback delay for reporting the selected mode to the transmitter. We assume a $4 \mathrm{~ms}$ (e.g., 4 subframes) feedback delay [48] corresponding to 4 subframes in the simulations. In this case, out of the 27 subframes used for data transmission, the transmitter employs the best mode from the previous data transmission session during the first 4 subframes, and then shifts to the best selected mode for this session for the remaining 23 subframes. We see that there is a negligible loss of less than $0.2 \mathrm{~dB}$ for narrow-band transmission, and around $0.4 \mathrm{~dB}$ loss for wide-band transmission, due to the feedback delay, hence the proposed scheme remains effective.

In order to study the impact of time lags in the presence of a larger number of antenna modes, we repeat the experiments for an MRA system with 20 active modes with 4 of them selected for training. We again use 500 subframes for initial training, implying that each mode is trained for only 25 times. After the initial training, 4 modes are selected for further training. Fig. 9 indicates the resulting MSE and ergodic Shannon capacity. It is again observed that the proposed CSI estimation/ prediction and mode selection methods can obtain most of the gains available with MRA systems, i.e., the performance is within $1 \mathrm{~dB}$ of the best attainable performance, and it provides around 4.5 dB SNR gain compared to the system using an arbitrarily picked mode.

\section{Impact of Mobility}

We next investigate the impact of user speed on the MRA system performance with the proposed channel estimation scheme (Figs. 10-12). As the user speeds increase, since the channel variations become more rapid, the periodic training parameters need to be reselected. For $5 \mathrm{~km} / \mathrm{h}$, suitable number of training and data symbols are found to be 12 and 36 , respectively, while for $20 \mathrm{~km} / \mathrm{h}, 6$ and 18 symbols provide the 


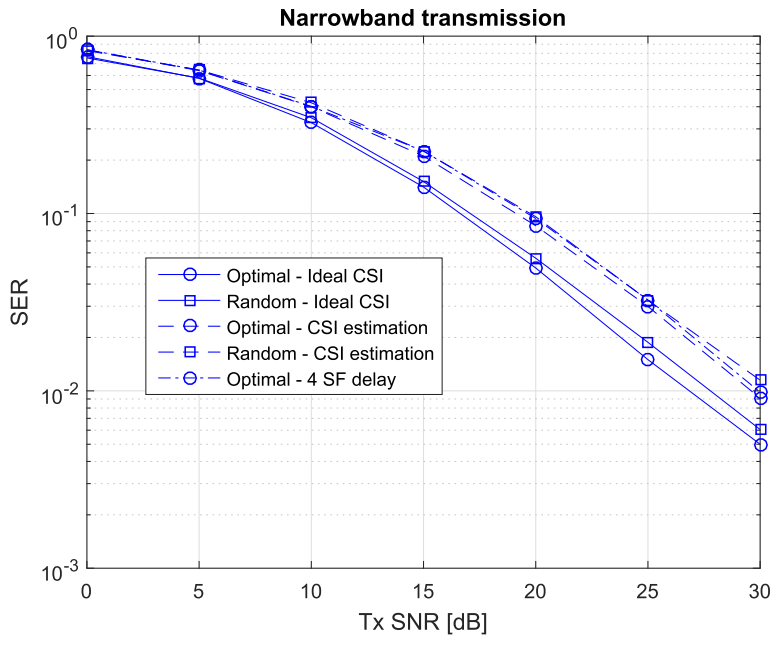

(a)

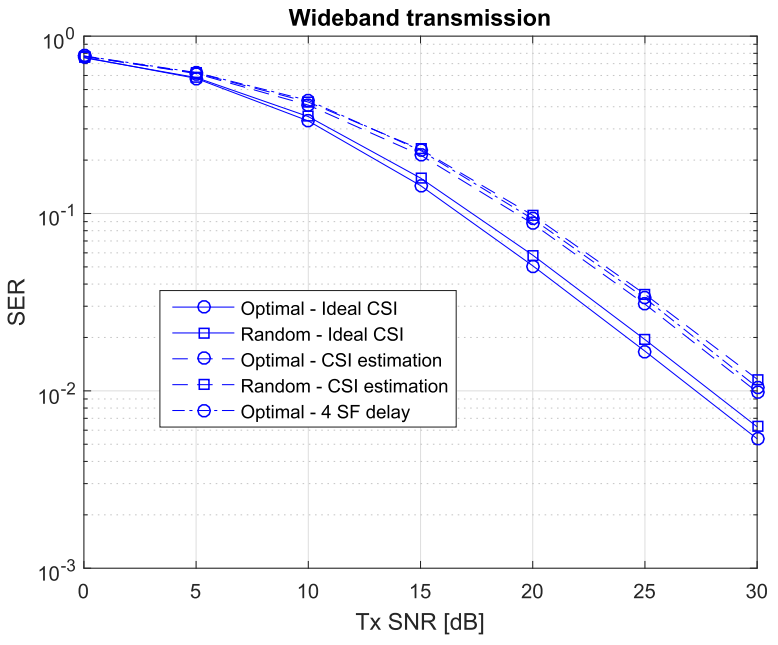

(b)

Fig. 12. Symbol error rate for $20 \mathrm{~km} / \mathrm{h}$ (a) narrowband (b) wideband transmission. For both cases, mode selection based on a single subcarrier is performed using (21).

best results. We observe that with the faster mode selection, the proposed scheme can track the best mode of operation and achieve a better performance compared to non-MRA systems. The channel capacity results are summarized in Fig. 10, and the resulting symbol error rates are depicted in Figs. 11 and 12. The symbol error rates with feedback delay of 4 subframes are also included in the figures. It is observed that at $5 \mathrm{~km} / \mathrm{h}$, the SNR loss is only about $0.5 \mathrm{~dB}$, while for the higher speed of 20 $\mathrm{km} / \mathrm{h}$, the SER is only slightly better than that for the system with a fixed antenna mode. Therefore, for channels with low coherence times, we suggest the use of MRAs for long-term performance improvements rather than extracting short-term gains.

\section{Discussion AND CONCLUding REMARKS}

We investigate the feasibility of pilot assisted channel estimation procedure for systems using MRAs. Exploiting the channel correlations resulting from different MRA modes, we propose a low-overhead CSI estimation procedure where only a subset of the modes of operation are employed for training. With the proposed approach, even with the presence of many antenna modes, the system can learn the modes of operation with a much smaller training subset. Using the developed techniques, one can approach within $1 \mathrm{~dB}$ of the achievable rates of MRA system with ideal CSI. In addition, the resulting symbol error rates indicate that one can achieve up to $3 \mathrm{~dB}$ SNR improvements compared to case assuming the availability of ideal CSI.

We develop a simple but realistic framework to show that with practical channel estimation approaches, one can achieve significant gains with MRAs, hence we argue that the use of MRAs in practical systems is promising as a future antenna candidate for $5 \mathrm{G}$ and beyond. In this work, we focus on a block MMSE based channel estimation/prediction procedure. Noting that the channel estimation for MRA antenna systems fits very well to the compressive sensing problem since we attempt to predict the channel for many different antenna modes using only a small subset of trained modes, a possible research direction is to develop compressed sensing based channel estimation and sparse Bayesian learning solutions for the mode selection scheme. An analytic framework associating the sparsity of multipath channel and radiation pattern space with the spatial correlations may be investigated towards that end. In addition, the relation between the configuration modes and the wireless channel can be further explored to implement MRAs that can create modes that are matched to the statistics of the scattering environment allowing for improved channel estimation along with superior performance.

\section{REFERENCES}

[1] J. T. Bernhard, Reconfigurable Antennas (Synthesis Lectures on Antennas and Propagation Series). San Rafael, CA, USA: Morgan \& Claypool, 2007.

[2] B. A. Cetiner, E. Akay, E. Sengul, and E. Ayanoglu, "A MIMO system with multifunctional reconfigurable antennas," IEEE Antennas Wireless Propag. Lett., vol. 5, no. 1, pp. 463-466, Dec. 2006.

[3] J. Rodriguez, Fundamentals of $5 G$ Mobile Networks. Hoboken, NJ, USA: Wiley, 2015.

[4] B. A. Cetiner, H. Jafarkhani, J.-Y. Qian, H. J. Yoo, A. Grau, and F. D. Flaviis, "Multifunctional reconfigurable MEMS integrated antennas for adaptive MIMO systems," IEEE Commun. Mag., vol. 42, no. 12, pp. 62-70, Dec. 2004.

[5] X. Yuan et al., "A parasitic layer-based reconfigurable antenna design by multi-objective optimization," IEEE Trans. Antennas Propag., vol. 60, no. 6, pp. 2690-2701, Jun. 2012.

[6] U. Afsheen, P. A. Martin, and P. J. Smith, "Space time state trellis codes for MIMO systems using reconfigurable antennas," IEEE Trans. Commun., vol. 63, no. 10, pp. 3660-3670, Oct. 2015.

[7] Y. Zhou, R. S. Adve, and S. V. Hum, "Design and evaluation of pattern reconfigurable antennas for MIMO applications," IEEE Trans. Antennas Propag., vol. 62, no. 3, pp. 1084-1092, Mar. 2014.

[8] C. G. Christodoulou, Y. Tawk, S. A. Lane, and S. R. Erwin, "Reconfigurable antennas for wireless and space applications," Proc. IEEE, vol. 100, no. 7, pp. 2250-2261, Jul. 2012.

[9] R. Mehmood and J. W. Wallace, "MIMO capacity enhancement using parasitic reconfigurable aperture antennas (RECAPs)," IEEE Trans. Antennas Propag., vol. 60, no. 2, pp. 665-673, Feb. 2012.

[10] Z. Li, Z. Du, and K. Gong, "Compact reconfigurable antenna array for adaptive MIMO systems," IEEE Antennas Wireless Propag. Lett., vol. 8 , pp. 1317-1320, 2009.

[11] J. D. Boerman and J. T. Bernhard, "Performance study of pattern reconfigurable antennas in MIMO communication systems," IEEE Trans. Antennas Propag., vol. 56, no. 1, pp. 231-236, Jan. 2008. 
[12] C. P. Sukumar, H. Eslami, A. M. Eltawil, and B. A. Cetiner, "Link performance improvement using reconfigurable multiantenna systems," IEEE Antennas Wireless Propag. Lett., vol. 8, pp. 873-876, 2009

[13] Z. Li, D. Rodrigo, L. Jofre, and B. A. Cetiner, "A new class of antenna array with a reconfigurable element factor," IEEE Trans. Antennas Propag., vol. 61, no. 4, pp. 1947-1955, Apr. 2013.

[14] H. Eslami et al., "Reduced overhead training for multi reconfigurable antennas with beam-tilting capability," IEEE Trans. Wireless Commun., vol. 9, no. 12, pp. 3810-3821, Dec. 2010.

[15] N. Gulati and K. R. Dandekar, "Learning state selection for reconfigurable antennas: A multi-armed bandit approach," IEEE Trans. Antennas Propag., vol. 62, no. 3, pp. 1027-1038, Mar. 2014.

[16] A. Grau, H. Jafarkhani, and F. D. Flaviis, "A reconfigurable multipleinput multiple-output communication system," IEEE Trans. Wireless Commun., vol. 7, no. 5, pp. 1719-1733, May 2008.

[17] R. Bhagavatula, C. Oestges, and R. W. Heath, "A new double-directional channel model including antenna patterns, array orientation, and depolarization," IEEE Trans. Veh. Technol., vol. 59, no. 5, pp. 2219-2231, Jun. 2010.

[18] M. T. Dao, V. A. Nguyen, Y. T. Im, S. O. Park, and G. Yoon, "3D polarized channel modeling and performance comparison of MIMO antenna configurations with different polarizations," IEEE Trans. Antennas Propag., vol. 59, no. 7, pp. 2672-2682, Jul. 2011

[19] A. Kalis, A. G. Kanatas, and C. B. Papadias, Eds., Parasitic Antenna Arrays for Wireless MIMO Systems. New York, NY, USA: Springer, 2013.

[20] C. Oestges and B. Clerckx, MIMO Wireless Communications: From Real-World Propagation to Space-Time Code Design. Orlando, FL, USA: Academic, 2007

[21] A. M. Sayeed, "Deconstructing multiantenna fading channels," IEEE Trans. Signal Process., vol. 50, no. 10, pp. 2563-2579, Oct. 2002.

[22] C. Oestges, V. Erceg, and A. J. Paulraj, "Propagation modeling of MIMO multipolarized fixed wireless channels," IEEE Trans. Veh. Technol., vol. 53, no. 3, pp. 644-654, May 2004.

[23] M. Steinbauer, A. F. Molisch, and E. Bonek, "The double-directional radio channel," IEEE Antennas Propag. Mag., vol. 43, no. 4, pp. 51-63, Aug. 2001.

[24] D. Rodrigo, B. A. Cetiner, and L. Jofre, "Frequency, radiation pattern and polarization reconfigurable antenna using a parasitic pixel layer," IEEE Trans. Antennas Propag., vol. 62, no. 6, pp. 3422-3427, Jun. 2014.

[25] W. L. Stutzman and G. A. Thiele, Antenna Theory and Design (Antenna Theory and Design). Hoboken, NJ, USA: Wiley, 2012.

[26] G. Durgin, Space-Time Wireless Channels (Prentice Hall Communications Engineering and Emerging Technologies Series). Englewood Cliffs, NJ, USA: Prentice-Hall, 2003.

[27] J. G. Proakis, Digital Communications (Electrical Engineering Series). New York, NY, USA: McGraw-Hill, 2001.

[28] P. N. Vasileiou, K. Maliatsos, E. D. Thomatos, and A. G. Kanatas, "Reconfigurable orthonormal basis patterns using ESPAR antennas," IEEE Antennas Wireless Propag. Lett., vol. 12, pp. 448-451, 2013.

[29] O. N. Alrabadi, J. Perruisseau-Carrier, and A. Kalis, "MIMO transmission using a single RF source: Theory and antenna design," IEEE Trans. Antennas Propag., vol. 60, no. 2, pp. 654-664, Feb. 2012.

[30] V. I. Barousis, A. G. Kanatas, and A. Kalis, "Beamspace-domain analysis of single-RF front-end MIMO systems," IEEE Trans. Veh. Technol., vol. 60, no. 3, pp. 1195-1199, Mar. 2011.

[31] V. Raghavan and A. M. Sayeed, "Sublinear capacity scaling laws for sparse MIMO channels," IEEE Trans. Inf. Theory, vol. 57, no. 1, pp. 345-364, Jan. 2011

[32] Y. Barbotin, A. Hormati, S. Rangan, and M. Vetterli, "Estimation of sparse MIMO channels with common support," IEEE Trans. Commun., vol. 60, no. 12, pp. 3705-3716, Dec. 2012.

[33] R. Prasad, C. R. Murthy, and B. D. Rao, "Joint channel estimation and data detection in MIMO-OFDM systems: A sparse Bayesian learning approach," IEEE Trans. Signal Process., vol. 63, no. 20, pp. 5369-5382, Oct. 2015.

[34] P. Kyösti et al., "IST-4-027756 WINNER II D1.1.2 V1.2: WINNER II channel models," Wireless World Initiative New Radio+ (WINNER+), Munich, Germany, Tech. Rep. D.1.1.2, Sep. 2007. [Online]. Available: http://projects.celtic-initiative.org/winner+/WINNER2Deliverables/D1.1.2.zip

[35] P. Heino et al., "CELTIC/CP5-026 D5.3: WINNER+ final channel models," CELTIC/WINNER Project, Tech. Rep., Jun. 2010 [Online]. Available: http://projects.celtic-initiative.org/winner+/ WINNER+\%20Deliverables/D5.3_v1.0.pdf
[36] L. Hentilä, P. Kyösti, M. Käske, M. Narandzic, and M. Alatossava. (2007). MATLAB Implementation of the WINNER Phase II Channel Model Ver1.1. [Online]. Available: fhttps://www.ist-winner.org/ phase2model.htmg

[37] K. Maliatsos and A. G. Kanatas, "Modifications of the IST-WINNER channel model for beamspace processing and parasitic arrays," in Proc. 7th Eur. Conf. Antennas Propag. (EuCAP), Apr. 2013, pp. 989-993.

[38] T. M. Duman and A. Ghrayeb, Coding for MIMO Communication Systems. Chichester, U.K.: Wiley, 2007.

[39] S. T. Chung and A. J. Goldsmith, "Degrees of freedom in adaptive modulation: A unified view," IEEE Trans. Commun., vol. 49, no. 9, pp. 1561-1571, Sep. 2001

[40] J. Meng and E.-H. Yang, "Constellation and rate selection in adaptive modulation and coding based on finite blocklength analysis and its application to LTE," IEEE Trans. Wireless Commun., vol. 13, no. 10, pp. 5496-5508, Oct. 2014.

[41] Z. B. Zabinsky, Stochastic Adaptive Search for Global Optimization (Nonconvex Optimization and Its Applications). New York, NY, USA: Springer, 2013.

[42] F. Dietrich, Robust Signal Processing for Wireless Communications (Foundations in Signal Processing, Communications and Networking). Heidelberg, Germany: Springer, 2007.

[43] "Evolved Universal Terrestrial Radio Access (E-UTRA); Further advancements for E-UTRA physical layer aspects," 3rd Generation Partnership Project (3GPP), Valbonne, France, Tech. Rep. TR 36.814 V9.0.0, Mar. 2010. [Online]. Available: http://www.3gpp.org/ftp/Specs/archive/36_series/36.814/36814-900.zip

[44] IEEE Standard for Local and Metropolitan Area Networks Part 16: Air Interface for Broadband Wireless Access Systems Amendment 3: Advanced Air Interface, IEEE Standard 802.16m-2011 (Amendment to IEEE Std 802.16-2009), May 2011, pp. 535-876.

[45] IEEE Standard for Information technology-Telecommunications and Information Exchange Between Systems Local and Metropolitan Area Networks-Specific Requirements Part 11: Wireless LAN Medium Access Control (MAC) and Physical Layer (PHY) Specifications, IEEE Standard 802.11-2012 (Revision of IEEE Std 802.11-2007), Mar. 2012, pp. 1669-1780.

[46] G. L. Stüber, J. R. Barry, S. W. McLaughlin, Y. Li, M. A. Ingram, and T. G. Pratt, "Broadband MIMO-OFDM wireless communications," Proc. IEEE, vol. 92, no. 2, pp. 271-294, Feb. 2004.

[47] ANSYS. (2016). ANSYS HFSS, Version 16.0. [Online]. Available: http://www.ansys.com

[48] E. Dahlman, S. Parkvall, and J. Skold, 4G: LTE/LTE-Advanced for Mobile Broadband. Oxford, U.K.: Elsevier, 2013.

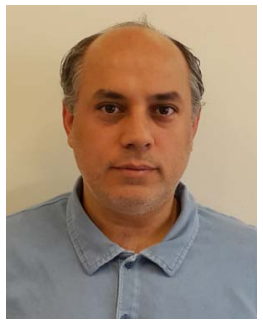

Israfil Bahceci (S'01-M'06) received the B.S. degree from Bilkent University, Turkey, in 1999, the M.S. degree from Arizona State University, Tempe, AZ, USA, in 2001, and the Ph.D. degree from the Georgia Institute of Technology, Atlanta, GA, USA, in 2005, all in electrical engineering. He was a Post-Doctoral Fellow with the University of Waterloo, ON, Canada, from 2005 to 2007. He was a Research and Development Engineer with Nortel Networks, Ottawa, ON, Canada, from 2007 to 2009, and Huawei Technologies, Ottawa, ON, Canada, from 2009 to 2011. He was with the TOBB University of Economics and Technology from 2011 to 2015. $\mathrm{He}$ has been a Research Scientist with the Department of Electrical and Computer Engineering, Utah State University, Logan, UT, USA, since 2015. His current research interests are in systems, with particular focus on communication and signal processing, including wireless and mobile communications, distributed estimation/detection, reconfigurable antenna systems for $5 \mathrm{G}$ and beyond systems.

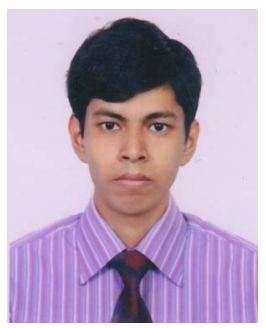

Mehedi Hasan received the B.Sc. degree in electrical engineering from the Bangladesh University of Engineering and Technology. He is currently pursuing the Ph.D. degree with the Department of Electrical and Computer Engineering, Utah State University, Logan, UT, USA. He was with Samsung Research and Development Institute Bangladesh from 2013 to 2015 . He is currently a Research Assistant with the Department of Electrical and Computer Engineering, Utah State University. His current research is focused on multifunctional reconfigurable antennas for use in 5G and beyond wireless systems. 


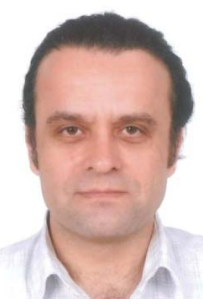

Tolga M. Duman (S'95-M'98-SM'03-F'11) received the B.S. degree from Bilkent University, Ankara, Turkey, in 1993, and the M.S. and Ph.D. degrees from Northeastern University, Boston, MA, USA, in 1995 and 1998, respectively, all in electrical engineering. He has been with the Electrical Engineering Department, Arizona State University, as an Assistant Professor from 1998 to 2004, an Associate Professor from 2004 to 2008, and a Professor from 2008 to 2015. $\mathrm{He}$ is a Professor of Electrical and Electronics Engineering Department, Bilkent University, and an Adjunct Professor with the School of ECE, Arizona State University. His current research interests are in systems, with particular focus on communication and signal processing, including wireless and mobile communications, coding/modulation, coding for wireless communications, data storage systems, and underwater acoustic communications.

Dr. Duman is a recipient of the National Science Foundation CAREER Award and the IEEE Third Millennium medal. He served as an Editor of the IEEE TRANSACTION ON WIRELESS COMMUNICATIONS from 2003 to 2008, the IEEE COMmunications Surveys AND Tutorials from 2002 to 2007, the IEEE TRANSACTION ON COMMUNICATIONS from 2007 to 2012, and the Physical Communication (Elsevier) from 2010 to 2016. $\mathrm{He}$ has been the coding and communication theory Area Editor of the IEEE TRANSACTION ON COMMUNICATIONS since 2011, an Editor of the IEEE TRANSACTION ON WIRELESS COMMUNICATION since 2016, and the Editor-in-Chief of the Physical Communication (Elsevier) since 2016.

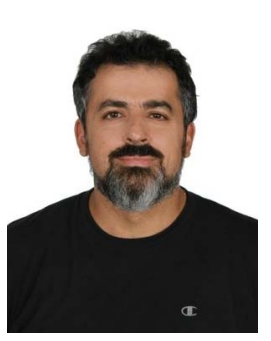

Bedri A. Cetiner (M'99) received the Ph.D. degree in electronics and communications engineering from Yildiz Technical University, Istanbul, in 1999. From 1999 to 2000, he was with the University of California at Los Angeles, Los Angeles, CA, USA, as a NATO Science Fellow. He then joined the Department of Electrical Engineering and Computer Science, University of California at Irvine, Irvine, CA, USA, where he was a Research Scientist from 2000 to 2004. From 2004 to 2007, he was an Assistant Professor with the Department of Space Science and Engineering, Morehead State University, Morehead, KY, USA. In 2007, he joined Utah State University. He is currently a Professor with the Department of Electrical Engineering, Utah State University. His research focuses on the applications of micronano technologies to a new class of micro-/millimeterwave circuits and systems, and intelligent wireless communications systems with an emphasis on the multifunctional reconfigurable antennas (MRA) and MRA arrays for use in cognitive multi-input multioutput systems. He is the principal inventor of nine patented technologies including microwave laminate compatible RF MEMS technology and MRA equipped MIMO systems. He is a member of the IEEE Antennas and Propagation, Microwave Theory and Techniques, and Communication Societies. 